\title{
The Use of Cyclosporine A in Rheumatology: A 2016 Comprehensive Review
}

\author{
Cecilia Beatrice Chighizola ${ }^{1,2,3}$, Voon H. Ong ${ }^{4}$, Pier Luigi Meroni ${ }^{1,2,5}$
}

${ }^{1}$ Allergology, Clinical Immunology and Rheumatology Unit, IRCCS Istituto Auxologico Italiano, piazzale Brescia 20, 20149 Milan, italy

2 Experimental laboratory of Immunological and Rheumatologic Researches, IRCCS Istituto Auxologico Italiano, Via Zucchi 18, 20095 Cusano Milanino, Milan, Italy

${ }^{3}$ Department of Clinical Sciences and Community Health, University of Milan, Via Festa del Perdono 7, 20122 Milan, Italy

${ }^{4}$ Centre for Rheumatology and Connective Tissue Diseases, Royal Free Hospital, London, UK

${ }^{5}$ Division of Rheumatology, ASST G. Pini, Pzza C Ferrari 1, 20122 Milan, Italy

\section{Corresponding author:}

Prof. Pier Luigi Meroni

IRCCS Istituto Auxologico Italiano

Via Zucchi 18

20095 Cusano Milanino, Milan, Italy

pierluigi.meroni@unimi.it

Tel: +3902619112554

Fax: +3902619113033 


\begin{abstract}
Cyclosporine A, an inhibitor of calcineurin, exerts an immunomodulator action interfering with $\mathrm{T}$ cell activation. Even though novel therapeutic tools have emerged, CyA still represents a suitable option in several clinical rheumatology settings. This is the case of refractory nephritis and cytopenias associated with systemic lupus erythematosus. Furthermore, CyA is a valued therapeutic tool in the management of uveitis and thrombophlebitis in course of Behçet's disease. Topical CyA has been proven to be beneficial in the dry eye of Sjogren's syndrome, whereas oral treatment with CyA can be considered for the severe complications of adult onset Still's disease. CyA provides a therapeutic option in psoriatic arthritis, being rather effective in skin disease. CyA is currently regarded as a second-line option for patients with inflammatory myopathies refractory to standard regimen. CyA is used even in paediatric rheumatology, in particular in the management of juvenile dermatomyositis and macrophage activation syndrome associated with systemic juvenile idiopathic arthritis. Importantly, CyA has been shown to suppress the replication of $\mathrm{HCV}$, and it can thus be safely prescribed to those patients with chronic hepatitis C. Noteworthy, CyA can be administered throughout the gestation course. Surely caution should be paid to CyA safety profile, in particular to its nephrotoxicity. Even though most evidence comes from small and uncontrolled studies with few randomized controlled trials, CyA should be still regarded as a valid therapeutic tool in 2016 rheumatology.
\end{abstract}

\title{
Key words
}

1. Cyclosporine

2. Rheumatology

3. Arthritides

4. Connective tissue diseases

5. Vasculitides

6. Hepatitis $\mathrm{C}$ virus 


\section{Introduction}

The earliest use of cyclosporine (CyA) in the field of rheumatology dates back to 1979, when the first trial in patients with inflammatory arthritides was conducted [1]. Since then, CyA has been used to manage several autoimmune conditions: thanks to its mechanism of action, this pharmacological compound is potentially effective in different clinical settings.

\section{Mechanism of action of cyclosporine A}

Initially developed as an anti-mycotic agent, CyA is an 11-aminoacid peptide originally isolated from a soil fungus. Its immunosuppressive properties have been described in the 70s, when it was first employed as an anti-rejection agent for organ transplantation. CyA is a pro-drug, which becomes active after complexing with cyclophillin, an intra-cytoplasmatic protein. The CyAcyclophillin complex in turn inhibits calcineurin, a phosphatase that mediates the pharmacological effects of CyA. Indeed, calcineurin induces the translocation into the nucleus of the nuclear factor of activated T cells (NF-AT), which acts as transcription factor for a number of pro-inflammatory cytokines, such as interleukin (IL)-2, IL-2 receptor, IL-4, interferon (IFN)- $\gamma$ and transforming growth factor- $\beta$ (TGF- $\beta$ ). The inhibition of IL-2 and IL-2 receptor, the two main stimulating pathways involved in $\mathrm{T}$ cell activation, accounts for the action of $\mathrm{CyA}$ on $\mathrm{T}$ cells: it impairs the activation of T helper (Th) cells, even though the T suppressor (Ts) subset may also be affected [2]. The pharmacological action of CyA is schematically presented in Figure $\mathbf{1}$.

\section{Pharmacokinetics of cyclosporine A}

Two formulations of CyA have been developed: the original one (Sandimmune) displays a poor bioavailability and larger inter and intra-subject pharmacokinetic variability; the most recent microemulsion (Neoral) offers a better bioavailability and a more predictable pharmacokinetics and dose linearity. Because of its lipophilic nature, plasma CyA is almost exclusively bound to lipoproteins, explaining why CyA serum concentrations might be affected by dietary fat intake. 
Indeed, raised serum lipid levels can increase CyA total body clearance: a higher serum CyA concentration results when the drug is administered before rather than after meals, accounting for higher clinical efficacy [2].

CyA displays a first-pass effect of $27 \%$ in the liver; the enterohepatic recirculation of CyA from the bile to the small intestine is responsible of its biphasic distribution. CyA half-life in serum ranges between 6 and 24 hours; its oral bioavailability and systemic clearance are controlled by the cytochrome P450 and the efflux p-glycoprotein pump, a trans-membrane transporter expressed in the gastrointestinal tract and in the liver. CyA metabolites are excreted primarily in the bile, with only $6 \%$ of the dose being excreted in the urine [2].

\section{Cyclosporine $\mathrm{A}$ in rheumatoid arthritis}

The rationale of CyA use in rheumatoid arthritis (RA) is supported by its mechanism of action: besides the inhibition of T cells, CyA has been demonstrated to suppress IL-17 production in vitro. This results in the impairment of the differentiation of Th17 cells, a distinct lineage of proinflammatory Th cells regarded as key effectors in RA pathogenesis [3]. The 2008 updated guidelines issued by the American College of Rheumatology (ACR) do not enlist CyA as a therapeutic option in the management of RA [4]. As a matter of facts, evidences about CyA efficacy in RA are not recent, and not so solid to support its routine use (Table 1). Over the years, CyA has been proven to be more effective than placebo in disease control [5-9], even at long term [10], delaying radiological progression [11-13]. Controlled trials have shown CyA to be as effective as parenteral gold [14,15], D-penicillamine [16], hydroxychloroquine (HCQ) and azathioprine (AZA) [17-19], but less effective than methotrexate (MTX) [20-22].

Combining CyA with HCQ, chloroquine or gold has been observed to convey no clear benefit [2326]. Conversely, association regimens comprising CyA plus MTX have been demonstrated to allow a greater rate of disease control as compared to CyA monotherapy [27-30] and CyA plus leflunomide (LEF) [31], using a step-up or a step-down approach. Clinical studies have investigated 
the combination regimen of CyA plus MTX to MTX alone, reporting a greater beneficial effect for the combo approach [32-34], a benefit not sustained on the long-term [35]. Consistently, MTX non responder patients have been shown to present a better disease course when randomized to MTX plus CyA than when receiving MTX plus placebo [36,37]. On the other hand, the addition of CyA to MTX and infliximab (IFX) has been proven to result only in a modest increase in the response [38,39], even though CyA plus MTX association regimen has been shown to be effective in maintaining disease remission after suspension of treatment with tumour necrosis factor (TNF) inhibitors in $62 \%$ of patients [40], avoiding even erosive progression at MRI [41]. A 2001 metaanalysis of randomized controlled trials (RCT) evaluating the optimal step-up strategy in patients with an incomplete response to MTX has found that the addition of CyA, etanercept (ETN), IFX or LEF results in a comparable ACR20 response at 24-30 weeks of combination therapy [42].

\section{Cyclosporine $A$ in psoriatic arthritis}

CyA has been shown to be more effective in the management of psoriatic arthritis (PsA) as compared to RA [43]. Such observation might be explained by the pivotal role played by IL-17 axis in PsA pathogenesis. Furthermore, CyA inhibits vascular endothelial growth factor (VEGF), a key mediator of the angiogenesis characteristic of PsA rather than RA synovium [44,45]. In the 2008 treatment guidelines for PsA, CyA is recommended in the management of moderate or severe peripheral arthritis, moderate to severe skin and nail disease [46]. Clinical improvement becomes usually evident after 3-4 weeks of treatment with CyA; response of both skin and joint involvement is dose-related, with the lowest optimal effective maintenance dose being around $3 \mathrm{mg} / \mathrm{Kg}$.

Observational studies have shown CyA to exert a beneficial effect in reducing peripheral (but not axial) joint involvement in patients with PsA [47-50]. Controlled trials have reported that CyA is as effective as MTX, but with a higher withdrawal rate [51], and more effective than sulfasalazine (SSZ) [52]. The association of CyA with MTX has been shown to lead to a significantly better joint disease control than MTX alone [53]; the addition of CyA to ETN in patients with PsA and 
uncontrolled cutaneous disease has emerged to be a safe and effective therapeutic option [54], being even better on psoriatic skin involvement than ETN plus MTX [55]. Response to CyA in PsA has been found to be persistent at two-year follow-up [56], controlling also the radiological damage [57]. Clinical studies on CyA in PsA are detailed in Table 2.

\section{Cyclosporine A in systemic lupus erythematosus}

In vivo, treatment with CyA has been found to prolong the life span of NZB/W F1 mice, reducing anti-DNA antibody titers [58-60]. The earliest experience with CyA treatment in patients with systemic lupus erythematosus (SLE) dates back to 1981; nowadays, CyA is not so commonly prescribed to lupus patients. In several small observational studies, CyA, alone or in combination with other agents, has been shown to reduce disease activity and improve immunological parameters such as anti-dsDNA titers and complement levels, allowing steroid tapering [6169](Table 3). In particular, CyA has been shown to be beneficial in some lupus manifestations, and it might be taken into account as therapeutic option in refractory lupus nephritis, skin disease and haematological involvement [70]. CyA efficacy in lupus nephritis is mediated not only by its immunosuppressive action but also its ability to stabilize the podocyte cytoskeleton by inhibiting dephosphorylation and degradation of synaptopodin, an actin-associated protein that regulates cell shape and motility and mediates podocyte foot processes. To note, synaptopodin downregulation and increased calcineurin activity are both associated with proteinuric glomerular diseases [71,72]. CyA is currently recommended in the management of lupus nephritis with persistent severe proteinuria, refractory to conventional treatment with corticosteroids and cytotoxic agents [70]. Indeed, according to still limited data, the association of CyA with corticosteroid therapy is effective in the treatment of class III, IV or V lupus nephritis. Most evidence suggests CyA efficacy in proliferative lupus nephritis, as supported by few case series [66,73-78] and uncontrolled trials $[79,80]$. In addition, CyA has been shown to be more beneficial than steroids alone [81] and as effective as AZA [82] and cyclophosphamide (CTX), both at short [83] and long term follow-up 
[84]. As a whole, it can be stated that CyA treatment in proliferative lupus nephritis displays a cumulative rate of complete or partial remission approaching 90\%, with an important antiproteinuric effect [85]. CyA has been proven to be effective also in the management of membranous lupus nephritis, even though evidence comes from small retrospective studies [86-89] and a single RCT comparing CyA to CTX as adjunctive treatments to steroids [90]. A 2014 metaanalysis considering CyA and tacrolimus has concluded that calcineurin inhibitors might be regarded as a reasonable alternative to CTX in the induction treatment of active lupus nephritis, with an higher rate of complete remission (relative risk $[R R]=1.56$ ) and a better response/total remission ratio $(\mathrm{RR}=1.23)$ [91]. On the other hand, when evaluating prescription of CyA in lupus nephritis, it should be mentioned that in a cross-sectional study on 64 patients with lupus nephritis, CyA treatment has emerged as a risk factor for arterial hypertension, conveying a odds ratio of 5.3 [92].

Haematological abnormalities, if relapsing or refractory to steroids, have been shown to respond well to CyA. This is the case of lupus-associated thrombocytopenia [93,94], haemolytic anaemia [95], aplastic anaemia [96], red cell aplasia [97-99] and haemophagocytic syndrome [100]. Unfortunately, literature is still limited to case reports and small case series.

CyA exerts an evident steroid-sparing effect in cutaneous lupus. Unfortunately, skin disease has not provided a primary outcome measure in most studies in SLE; however, cutaneous manifestations have been reported to improve upon CyA treatment $[65,68]$. In addition, there are case reports of CyA efficacy in subacute cutaneous lupus erythematosus (LE) [101] and LE profundus [102], but not in discoid LE [103,104].

\section{Cyclosporine A in systemic sclerosis}

In vitro, CyA has been observed to inhibit the pro-fibrotic effects induced by TGF- $\beta$ and IL-4 in fibroblasts, leading to a decreased synthesis of collagen [105-107]. Although there is a rationale for its use, data in support of the efficacy of CyA in systemic sclerosis (SSc) are not solid (Table 4). 
CyA has been reported to significantly improve skin thickening in some, but not all, scleroderma patients; available evidence comes from small, short-term studies [69,108-111]. In particular, an improvement of oesophageal motor function has emerged with CyA treatment [111,112]; conversely, CyA has been described not to affect scleroderma-related internal organ involvement, in particular lung and cardiac disease [108]. There is a single report of the benefit of CyA in interstitial lung disease (ILD): one patient with diffuse scleroderma received CyA as maintenance therapy after CTX pulses, with evidences of stable disease at two-year clinical and radiological follow-up [113]. It is thus not surprising that the guidelines for scleroderma treatment published by the European League Against Rheumatism Scleroderma Trials and Research Group have not recommend CyA in SSc, highlighting the need of larger prospective studies [114]. It should be noted that CyA use in SSc is limited by its nephrotoxicity: indeed, CyA might affect renal function through a direct toxic action on the nephron or through an impairment of renal haemodynamic homeostasis. Consequently, CyA may not only worsen arterial hypertension, but also precipitate acute renal failure. In the past, CyA has even been proposed as risk factor for scleroderma renal crisis, but such association has not been confirmed in later studies [115].

\section{Cyclosporine A in inflammatory myopathies}

In the setting of inflammatory myopathies (polymyositis [PM] and dermatomyositis [DM]), CyA is currently regarded as a second-line option to be reserved to patients with disease refractory to standard regimen, which includes high-dose prednisone plus MTX or AZA.

Few trials have investigated CyA efficacy in inflammatory myopathies (Table 5). In a prospective controlled study, 10 DM patients receiving CyA have achieved a prompt remission; therapeutic failure occurred in $10 \%$ of cases as compared to $9 \%$ in those treated with standard regimen [116]. In addition, in a RCT on 36 patients (20 with DM, 16 with PM), CyA has been shown to be as effective as MTX [117]. In literature there are reports about CyA efficacy in managing DMassociated oesophageal involvement [118]. A growing body of evidence suggests the beneficial 
effect of CyA in the management of ILD [119-123], also in cases resistant to steroid treatment $[124,125]$ and those with an acute onset [126]. In particular, disease stabilization -or even an increase greater than $10 \%$ in forced vital capacity- has been reported in all series. CyA has also been compared to CTX in the management of PM/DM-ILD: in a cohort of 15 anti-Jo1-positive patients with lung involvement, high resolution scan of the chest at 12-month follow-up has revealed a disease worsening in $71 \%$ of patients treated with CyA as compared to $50 \%$ of those receiving CTX, with no differences in CT changes [127].

\section{Cyclosporine A in systemic vasculitides}

CyA is not enlisted as a therapeutic option in the current guidelines for the management of antineutrophil cytoplasmic antibody (ANCA)-associated vasculitides: the evidence of CyA efficacy in these clinical settings is rather scarce (Table 6). There are only anecdotal reports of patients with refractory granulomatosis with polyangiitis (GPA, formerly known as Wegener granulomatosis) successfully treated with CyA. In particular, CyA has led to the improvement of renal function and to the complete resolution of lung lesions [128-130], while in other cases CyA has not been effective in achieving disease remission [131]. A single report of CyA in eosinophilic granulomatosis with polyangiitis (EGP, formerly known as Churg-Strauss syndrome) has been published: a patient with EGP resistant to treatment with pulse intravenous CTX responded well to CyA therapy [132].

Similarly, the burden of evidence suggesting CyA efficacy in large vessels vasculitides is poor. In a RCT, CyA has been evaluated as steroid sparing agent in 22 patients with giant cells arteritis. However, the addition of CyA to corticosteroid treatment does not appear to be beneficial [133135]. CyA has been successfully used in the management of patients with Takayasu's arteritis $[134,136]$, in particular in those subjects with severe pyoderma gangrenosum $[137,138]$. 


\section{Cyclosporine A in Behçet's disease}

The efficacy of CyA in Behçet's Disease (BD) is supported by the inhibition of IL-1, a keycytokine in disease pathogenesis [139]. Given the wide heterogeneity in the clinical manifestations of $\mathrm{BD}$, the therapeutic approaches might be rather diverse, depending upon the presenting symptoms. Even though CyA has been shown to be effective in almost all clinical manifestations of $\mathrm{BD}$, its use is currently recommended in the management of refractory ocular involvement and thrombophlebitis [140] (Table 7). In particular, refractory eye disease provides the main setting for CyA prescription in BD. Indeed, CyA is the agent of choice, alternatively to IFX, in the management of eye involvement not responsive to standard treatment, to be prescribed in combination with AZA and corticosteroids [140]. This recommendation is supported by three RCT concordantly showing CyA efficacy in ocular involvement [141-143], even though to a lower extent than IFX [144]. To note, CyA is rapidly effective in acute uveitis, although the beneficial effects seem to be not sustained in the long term $[145,146]$.

CyA is currently recommended also in the management of thrombophlebitis, based on an open trial showing complete resolution of venous insufficiency with no recurrences over a two-month followup in seven patients [147]. In addition, CyA has been demonstrated to be effective in case of ulcers, oral as well as genital, and erythema nodosum, being superior to colchicine $[143,148]$. However, CyA is regarded as fourth-line agent because of its toxicity profile. In particular, because of its neurotoxicity, CyA should be not prescribed to BD patients with central nervous system involvement, unless necessary to manage intraocular inflammation [140]. CyA neurologic side effects in BD have been confirmed in three case-controlled studies [149-151].

\section{Cyclosporine A in adult onset Still's disease}

Corticosteroids still provide the mainstay of treatment of adult onset Still's disease, and MTX is the first-line steroid-sparing option. Targeted biologic agents, mainly IL-1 antagonists and TNF- $\alpha$ inhibitors, are reserved to refractory cases [152]. Even though there are reports of CyA efficacy as 
steroid sparing agent with a rapid effect and a low recurrence rate [153], its use in adult onset Still's disease is currently limited to some of the severe manifestations of Still's disease [154]. Indeed, CyA, alone or in combination with other pharmacological tools, might be considered as an option in the management of disseminated intravascular coagulation [155,156], macrophage activation syndrome (MAS) [157,158], acquired amegakariocytic thrombocytopenia [159] and severe hepatic failure [160]. Details of studies investigating CyA in adult onset Still's disease are presented in

\section{Table 8.}

\section{Cyclosporine A in Sjogren's syndrome}

Ex vivo studies have showed that topical CyA therapy induces a reduction in the number of activated T lymphocytes and apoptotic cells in conjunctival biopsies, a decreased expression of proinflammatory cytokines and a raise in goblet cell epithelial density [161-163]. Topical CyA is currently recommended in the management of dry eye (symptoms severity of level 2) associated with Sjogren's syndrome, at the dose of one drop in each eye twice daily [164]. Indeed, almost all available RCT have reported topical CyA to improve symptoms and signs of ocular sicca syndrome as compared to placebo or alternative treatment [165-169]. No clear dose-response relationship could be identified, but CyA $0.05 \%$ and $0.1 \%$ emulsions are the preparations most commonly prescribed.

Oral CyA has been evaluated in a single double-blind study conducted in a cohort of 20 patients, with a significant improvement of xerostomia and no significant differences in the Schirmer test score $[170,171]$. Details of studies investigating CyA in Sjogren's syndrome are presented in Table 9.

\section{Cyclosporine A in paediatric rheumatology}

CyA is currently used even in the field of paediatric rheumatology, with two particular indications: juvenile DM (JDM) and systemic juvenile idiopathic arthritis (sJIA). Few small retrospective 
studies and case series have investigated the beneficial effects of CyA in JDM. Even though corticosteroids are the only agents currently approved by the US Food and Drug Administration for JDM, many patients require additional immunosuppressive medications. In JDM patients unresponsive to corticosteroids or immunosuppressive drugs (MTX, AZA or CTX), CyA treatment has resulted in the improvement of muscle strength, allowing steroid tapering [172-174]. None of the immunosuppressive drugs have been yet evaluated in controlled trials in children; a 5-year, phase-III, single-blind, randomized, controlled trial is currently on-going. It envisages 3 treatment arms: i) prednisone; ii) prednisone plus MTX and iii) prednisone plus CyA. This international trial involves 185 partners from 46 countries, coordinated by PRINTO (Pediatric Rheumatology International Trials Organisation) and conducted in children with newly diagnosed JDM. An analysis of primary outcome measures after 6 months of treatment has showed that time to inactive disease is significantly shorter and time to major therapeutic changes significantly longer in the combination groups compared to prednisone alone. The highest rate of adverse events has been registered in the group treated with CyA (51\%) compared to MTX (28\%) or prednisone alone $(21 \%)[175]$.

The guidelines issued by ACR in 2013 recommends CyA as third-line treatment for sJIA children with active systemic features and a physician global assessment higher than 5 [176]. This recommendation is based upon prospective and retrospective series that have showed that CyA is effective in treatment of sJIA, leading to improvement in laboratory variables, joint counts, joint swelling, and morning stiffness in some -but not all- children, even if the improvement is not sustained at long term $[173,174,177,178]$. CyA has been evaluated even as second-line tool in sJIA, showing a good efficacy when associated with MTX [179]. CyA is particularly effective in MAS, a potentially life-threatening complication occurring in $7-13 \%$ of sJIA patients. Evidence of CyA efficacy in MAS associated with sJIA relies upon observational studies and case series (Table 10). CyA has been proven to result in clinical improvement in most patients, being particularly effective 
in the management of systemic symptoms [180-187]. Importantly, CyA has been reported lifesaving in serious cases of steroid resistant MAS [180].

\section{Cyclosporine $\mathrm{A}$ and hepatitis $\mathrm{C}$ virus}

CyA has been reported to suppress the replication of hepatitis $\mathrm{C}$ virus (HCV) both in vitro and in vivo, in a dose-dependent manner [188]. Such effect is mediated by the inhibition of cyclophillin B [189]. The maximum inhibitory effect exerted by CyA is similar to that of IFN- $\alpha$; to note, these two agents are certainly additive and possibly synergistic [190]. In addition CyA displays an antifibrotic effect inhibiting collagen production and increasing collagenase activity in hepatic stellate cells [191]. These data account for the safety and efficacy of CyA in the treatment of patients with autoimmune disorders and concomitant chronic HCV infection, as documented by some case-series with improvement of liver function and decreased viral load [192,193]. Furthermore, CyA safety in HCV positive RA patients has been evaluated in combination with anti-TNF- $\alpha$ agents, without any significant adverse event [194].

\section{Cyclosporine A and pregnancy}

CyA, being highly lipophilic, crosses the placenta to achieve in the fetal circulation a 10-50\% concentration of that in maternal plasma. Animal studies have not shown increased malformation rates, but in utero exposure to $\mathrm{CyA}$ in rabbits induces a nephron reduction leading to systemic hypertension and progressive chronic renal insufficiency in adulthood [195]. These in vivo observations explain why the FDA currently classifies CyA in the pregnancy risk category C.

Data on CyA in pregnancies are mostly derived from registries of pregnant transplant recipients, with more than 5000 observed pregnancies. Most studies of pregnancies exposed to CyA have concluded that there is no evidence for teratogenicity. On the other hand, an increase in premature delivery and low birth weight has been described, with $56 \%$ and $43 \%$ prevalence rates in a metaanalysis of 15 studies [196]. It is still not clear whether prematurity and low birth weight are 
actually due to CyA therapy or rather related to the underlying disease. Conversely, experience in pregnant patients with autoimmune disease is limited to case reports and case series accounting for about 50 pregnancies. No increase of congenital malformations or any particular malformation pattern has emerged; however, a higher incidence of low birth weight and prematurity has been described in CyA-treated women with autoimmune diseases as compared to the general population, even though to a lesser extent than in transplanted women [197]. A 12-year follow-up of 175 children exposed to CyA in utero has showed a $16 \%$ incidence of mental developmental delay, which might be attributed to the high incidence of prematurity [198]. Other studies have shown that maternal treatment with CyA during pregnancy does not permanently affect the foetal immune system $[199,200]$. As a whole, CyA is considered to be safe during pregnancy, therefore treatment can be continued at the lowest effective dose throughout the entire gestation. A periodic control of maternal blood pressure and renal function should be performed on a regular basis.

\section{Cyclosporine A and breastfeeding}

CyA is excreted in breast milk, with a wide variation in the milk-to-maternal serum concentration ratio, depending on the time of sampling and maternal dose. To date, evidence on the safety of breastfeeding during CyA therapy is limited to small case series and case reports $[201,202]$. No adverse events related to maternal CyA treatment during lactation have been described, with CyA being undetectable in almost all cases. Even though these data are reassuring, evidence is limited and still inconclusive. To date, breastfeeding during CyA treatment is not recommended because of theoretical risks of immunosuppressive effects in the neonate [203].

\section{Side effects}

In a study involving 154 patients with RA, a dose-related decrease in glomerular filtration rate with corresponding increase in serum creatinine has emerged as the most frequently reported side-effect of CyA (48\%); after drug discontinuation, creatinine returns within $15 \%$ of baseline in almost all 
cases [204]. There is a wide variety in the incidence of new-onset arterial hypertension, which ranges from 0 to $57 \%$ across available studies. Gastrointestinal symptoms, including nausea, abdominal pain, diarrhoea and vomiting, have been described in less than 5\%. Hepatotoxicity (presenting as elevation of hepatic enzymes and bilirubin) usually occurs at higher dosages than those used in rheumatology. Neurologic side effects of CyA include headache, tremor, seizure psychosis, paraesthesias, and sleep disturbances; paraesthesias and tremor can occur in up to $40 \%$ of patients. Gingival hyperplasia has been reported in up to $30 \%$ of patients on CyA; cutaneous side effects comprise hypertrichosis, epidermal cyst, keratosis pilaris, and sebaceous hyperplasia. Hypertrichosis is rather frequent, presenting in up to $60 \%$ of patients. Hyperlipidemia, in particular hypertriglyceridemia, can present in up to $15 \%$ of patients [205,206]. Based on epidemiologic analyses, FDA warns about a relationship between CyA and malignant lymphomas among RA patients and about an increased hazard of skin and lymphoproliferative malignancies among patients with psoriasis receiving CyA, even though the relative risk is similar to subjects treated with other immunosuppressive agents [207]. To date, there is no evidence that CyA predisposes to solid malignancies; large controlled studies have even suggested an immunoprotective action of CyA with a decreased OR for breast and colon neoplasias [204]. Table 11 reports the toxicity profile of CyA in RA patients.

\section{Drug interactions}

Table 12 reports the pharmacological compounds interfering with CyA pharmacokinetics. As CyA is mainly metabolized by cytochrome P450, drugs that induce hepatic enzyme metabolism lower CyA concentrations, while drugs that inhibit hepatic metabolism raise CyA blood concentrations. Older age, concomitant renal failure, arterial hypertension and dehydration might also contribute to enhance the nephrotoxicity of CyA [206]. 


\section{Drug safety monitoring}

Due to CyA nephrotoxicity, serum creatinine and blood pressure should be checked on at least two occasions to obtain a baseline value prior to therapy. Both creatinine and blood pressure should be monitored fortnightly during the first 3 months of treatment and monthly thereafter. Dose reduction of $0.5-0.75 \mathrm{mg} / \mathrm{kg} / \mathrm{day}$ is warranted if serum creatinine increases above baseline by $30 \%$-even if still in the normal range- or if uncontrolled hypertension supervenes. When creatinine increases by more than $50 \%$ of baseline, the daily dose dosage should be reduced by $50 \%$; if this reduction does not affect the creatinine value, the drug should be discontinued. If arterial hypertension develops, the use of anti-hypertensive agents should be considered, avoiding potassium-sparing diuretics because of the risk of hyperkaliemia. In case of hyperlipidemia, a lipid-lowering diet should be commenced and a lipid-lowering agent introduced. Since CyA decreases the clearance of statins, rhabdomyolisis may occur, thus warranting strict vigilance. Given that in patients with autoimmune diseases a poor correlation between CyA blood levels and its clinical effect has been noted, the monitoring of CyA plasma concentration is not routinely required [205,206].

\section{Conclusion}

CyA exerts a valid immunomodulator action, being effective in the treatment of many autoimmune diseases. Even though novel therapeutic tools have emerged over the recent years, CyA still represents a suitable option in several clinical settings:

a) In SLE, refractory lupus nephritis and cytopenias have been reported to respond well to CyA;

b) In PsA, CyA can be listed as a therapeutic option, being rather effective in cutaneous disease;

c) In BD, CyA is a valued treatment tool in the management of uveitis and thrombophlebitis;

d) In adult onset Still's disease, treatment with CyA can be considered for the most severe complications such as disseminated intravascular coagulation and MAS; 
e) In dry eye disease, topical CyA has been proven to be beneficial;

f) In inflammatory myopathies, CyA is currently regarded as a second-line option to be reserved to patients with disease refractory to standard regimen;

g) In paediatric rheumatology, CyA is used in the management of JDM and MAS associated with sJIA.

Conversely, CyA seems to exert an overall poor beneficial effect in SSc, Sjogren's syndrome and systemic vasculitides. CyA can be considered as an anchor drug in particular clinical situations:

a) HCV-positive patients: CyA has been shown to suppress the replication of $\mathrm{HCV}$, and it can thus be administered to those patients with $\mathrm{HCV}$;

b) Pregnancy: CyA can be safely prescribed throughout the gestation course, providing a pharmacological option in pregnant women with an active autoimmune disease.

Surely caution should be paid to CyA safety profile, in particular because of its nephrotoxicity: prescribing this drug should be avoided in patients with a raised baseline creatinine level and uncontrolled hypertension. Unfortunately, most of available evidence about CyA efficacy in rheumatology comes from small and uncontrolled studies, with very few RCT. Nevertheless, available data are encouraging, supporting CyA as a corticosteroid-sparing action in many rheumatologic conditions: despite being regarded as an "out-of-fashion" drug, CyA should be still part of therapeutic armamentarium of rheumatologists, even in 2016.

\section{Acknowledgements}

This work was supported in part by Ricerca Corrente IRCCS Istituto Auxologico Italiano to PLM. 


\section{Figure 1. Mechanism of action of cyclosporine A.}

$\mathrm{CyA}=$ cyclosporine $\mathrm{A} ; \mathrm{Ca}^{2+}=$ calcium; $\mathrm{NF}-\mathrm{ATc}-\mathrm{P}=$ phosphorylated cytoplasmic nuclear factor of activated T-cells; NF-ATc $=$ cytoplasmic nuclear factor of activated T-cells; NF-ATn $=$ nuclear nuclear factor of activated T-cells; IL-2= interleukin 2. 
Table 1. Clinical studies on cyclosporine A in rheumatoid arthritis.

\begin{tabular}{|c|c|c|c|c|c|c|c|}
\hline Author [Ref] & Year & $\begin{array}{l}\text { Study } \\
\text { design }\end{array}$ & CyA dose & Follow-up & $\begin{array}{c}\mathrm{N} \text { of } \\
\text { patients }\end{array}$ & Treatment groups & Outcome \\
\hline Dougados [5] & 1987 & $\mathrm{CS}$ & $5 \mathrm{mg} / \mathrm{Kg} /$ day & 12 months & 12 & CyA & Good clinical efficacy \\
\hline $\begin{array}{l}\text { Førre } \\
{[19]}\end{array}$ & 1987 & $\mathrm{CC}$ & $10 \mathrm{mg} / \mathrm{Kg} /$ day & 26 weeks & 24 & $\begin{array}{l}\text { CyA } \\
\text { AZA }\end{array}$ & $\begin{array}{l}\text { CyA group: improvement in } \\
\text { Ritchie index, PI joint } \\
\text { circumferences, } 50 \text {-foot walk } \\
\text { time, grip strength } \\
\text { AZA group: improvement in grip } \\
\text { strength }\end{array}$ \\
\hline Weinblatt [7] & 1987 & $\mathrm{CS}$ & $6 \mathrm{mg} / \mathrm{Kg} /$ day & 24 weeks & 10 & CyA & $\begin{array}{l}\text { Significant improvement in joint } \\
\text { pain, joint swelling indexes, } \\
\text { patient and physician assessments }\end{array}$ \\
\hline Dougados [6] & 1989 & $\mathrm{CS}$ & $5 \mathrm{mg} / \mathrm{Kg} /$ day & 12 months & 49 & CyA & $\begin{array}{l}\text { CyA discontinued in } 32 \text { patients } \\
\text { because of inefficacy or side- } \\
\text { effects } \\
\text { In } 17 \text { patients still receiving CyA, } \\
\text { significant clinical improvement } \\
\text { persisted at } 12 \text {-month evaluation }\end{array}$ \\
\hline Tugwell [9] & 1990 & $\mathrm{RCT}$ & $2.5 \mathrm{mg} / \mathrm{Kg} /$ day & 6 months & 144 & $\begin{array}{c}\text { CyA } \\
\text { Placebo }\end{array}$ & $\begin{array}{l}\text { Significant improvement in CyA } \\
\text { group compared to placebo group } \\
\text { in joint pain, active joint count, } \\
\text { functional status. }\end{array}$ \\
\hline van Rijthoven [10] & 1991 & $\mathrm{RCT}$ & $5 \mathrm{mg} / \mathrm{Kg} /$ day & 24 weeks & 92 & $\begin{array}{c}\text { CyA } \\
\text { D-penicillamine }\end{array}$ & $\begin{array}{l}\text { CyA and D-penicillamine equally } \\
\text { effective }\end{array}$ \\
\hline $\begin{array}{l}\text { Ahern } \\
{[18]}\end{array}$ & 1991 & $\mathrm{RCT}$ & $4.2 \mathrm{mg} / \mathrm{Kg} /$ day & 6 months & 52 & $\begin{array}{l}\text { CyA } \\
\text { AZA }\end{array}$ & CyA and AZA equally effective \\
\hline van Rijthoven [16] & 1991 & $\mathrm{CS}$ & $5 \mathrm{mg} / \mathrm{Kg} /$ day & 6 months & 16 & CyA & $\begin{array}{l}\text { CyA discontinued in } 6 \text { patients } \\
\text { because of inefficacy or side- } \\
\text { effects } \\
\text { In } 12 \text { patients still receiving CyA, } \\
\text { significant clinical improvement } \\
\text { in all considered variables. }\end{array}$ \\
\hline
\end{tabular}




\begin{tabular}{|c|c|c|c|c|c|c|c|}
\hline $\begin{array}{l}\text { Madhok } \\
{[8]}\end{array}$ & 1991 & $\mathrm{CS}$ & $5 \mathrm{mg} / \mathrm{Kg} /$ day & 24 months & 20 & CyA & $\begin{array}{l}\text { Significant improvement in } \\
\text { Ritchie index, joint pain, CRP, } \\
\text { joint function }\end{array}$ \\
\hline $\begin{array}{l}\text { Bensen } \\
{[28]}\end{array}$ & 1994 & $\mathrm{CC}$ & $2.5 \mathrm{mg} / \mathrm{Kg} /$ day & 12 months & 40 & $\begin{array}{l}\text { CyA + gold } \\
\text { CyA + MTX }\end{array}$ & $\begin{array}{l}\text { Increased efficacy with the } \\
\text { addition of CyA to gold or MTX }\end{array}$ \\
\hline $\begin{array}{l}\text { Kruger } \\
{[17]}\end{array}$ & 1994 & $\mathrm{RCT}$ & $5 \mathrm{mg} / \mathrm{Kg} /$ day & 6 months & 117 & $\begin{array}{l}\text { CyA } \\
\text { AZA }\end{array}$ & CyA and AZA equally effective \\
\hline $\begin{array}{l}\text { Førre } \\
{[13]}\end{array}$ & 1994 & $\mathrm{RCT}$ & $5 \mathrm{mg} / \mathrm{Kg} /$ day & 48 weeks & 122 & $\begin{array}{l}\text { CyA } \\
\text { Placebo }\end{array}$ & $\begin{array}{l}\text { Slower radiologic progression in } \\
\text { CyA group }\end{array}$ \\
\hline $\begin{array}{l}\text { Tugwell } \\
{[30]}\end{array}$ & 1995 & $\mathrm{RCT}$ & $\begin{array}{c}2.5-5 \\
\mathrm{mg} / \mathrm{Kg} / \mathrm{day}\end{array}$ & 6 months & 148 & $\begin{array}{c}\text { CyA + MTX } \\
\text { MTX + placebo }\end{array}$ & $\begin{array}{c}\text { CyA + MTX more effective than } \\
\text { MTX + placebo in severe RA }\end{array}$ \\
\hline $\begin{array}{l}\text { Salaffi } \\
\text { [29] }\end{array}$ & 1996 & $\mathrm{CC}$ & $2.5 \mathrm{mg} / \mathrm{Kg} /$ day & 6 months & 28 & $\begin{array}{l}\mathrm{CyA}+\mathrm{HCQ} \\
\mathrm{CyA}+\mathrm{MTX}\end{array}$ & $\begin{array}{l}\text { CyA + MTX more effective and } \\
\text { with more side-effects than CyA } \\
+ \text { HCQ }\end{array}$ \\
\hline $\begin{array}{l}\text { Pasero } \\
{[12]}\end{array}$ & 1996 & $\mathrm{RCT}$ & $3 \mathrm{mg} / \mathrm{Kg} /$ day & 12 months & 361 & $\begin{array}{c}\text { CyA } \\
\text { Antimalarials } \\
\text { Gold } \\
\text { SSZ }\end{array}$ & $\begin{array}{c}\text { Slower radiologic progression in } \\
\text { CyA group compared to DMARD } \\
\text { group }\end{array}$ \\
\hline $\begin{array}{l}\text { Bendix } \\
{[25]}\end{array}$ & 1996 & $\mathrm{RCT}$ & $2.5 \mathrm{mg} / \mathrm{Kg} /$ day & 6 months & 40 & $\begin{array}{c}\text { CyA + Gold } \\
\text { Gold + placebo }\end{array}$ & $\begin{array}{c}\text { CyA }+ \text { gold as effective as gold }+ \\
\text { placebo }\end{array}$ \\
\hline $\begin{array}{l}\text { Stein } \\
{[36]}\end{array}$ & 1997 & $\mathrm{CC}$ & $2.5 \mathrm{mg} / \mathrm{Kg} /$ day & 48 weeks & 92 & $\mathrm{CyA}+\mathrm{MTX}$ & $\begin{array}{l}\text { Clinical improvement with the } \\
\text { addition of CyA to MTX }\end{array}$ \\
\hline $\begin{array}{l}\text { Van de Borne } \\
{[26]}\end{array}$ & 1998 & $\mathrm{RCT}$ & $\begin{array}{c}1.2 .5 \\
\mathrm{mg} / \mathrm{Kg} / \mathrm{day}\end{array}$ & 24 weeks & 88 & $\begin{array}{c}\text { CyA + chloroquine } \\
\text { Chloroquine + placebo }\end{array}$ & $\begin{array}{l}\text { CyA + chloroquine moderately } \\
\text { more effective than chloroquine }+ \\
\text { placebo }\end{array}$ \\
\hline $\begin{array}{l}\text { Zeidler } \\
{[14]}\end{array}$ & 1998 & $\mathrm{RCT}$ & 3 mg/Kg/day & 18 months & 375 & $\begin{array}{l}\text { CyA } \\
\text { Gold }\end{array}$ & CyA and gold equally effective \\
\hline
\end{tabular}




\begin{tabular}{|c|c|c|c|c|c|c|c|}
\hline $\begin{array}{l}\text { Drosos } \\
{[21]}\end{array}$ & 1998 & RCT & $3 \mathrm{mg} / \mathrm{Kg} /$ day & 24 months & 103 & $\begin{array}{l}\text { CyA } \\
\text { MTX }\end{array}$ & CyA and MTX equally effective \\
\hline $\begin{array}{l}\text { Proudman } \\
\text { [208] }\end{array}$ & 2000 & RCT & $1.5 \mathrm{mg} / \mathrm{Kg} /$ day & 48 weeks & 82 & $\begin{array}{c}\text { CyA + MTX + i.a. steroids } \\
\text { SSZ }\end{array}$ & $\begin{array}{l}\text { Similar remission rate in the CyA } \\
+ \text { MTX + steroid group and in the } \\
\text { SSZ group }\end{array}$ \\
\hline $\begin{array}{l}\text { Drosos } \\
{[11]}\end{array}$ & 2000 & $\mathrm{RCT}$ & $3 \mathrm{mg} / \mathrm{Kg} /$ day & 24 months & 103 & $\begin{array}{c}\mathrm{CyA}+\mathrm{PDN} \\
\mathrm{MTX}+\mathrm{PDN}\end{array}$ & $\begin{array}{c}\text { CyA and MTX equally effective } \\
\text { in preventing radiological } \\
\text { progression }\end{array}$ \\
\hline $\begin{array}{l}\text { Ferraccioli } \\
{[37]}\end{array}$ & 2002 & $\mathrm{CC}$ & $3 \mathrm{mg} / \mathrm{Kg} /$ day & 18 months & 126 & $\begin{array}{c}\text { CyA, then CyA + MTX } \\
\text { MTX, then CyA + MTX } \\
\text { SSZ } \\
\text { CyA + MTX + SSZ }\end{array}$ & $\begin{array}{c}\text { CyA + MTX + SSZ effective in } \\
\text { control disease activity }\end{array}$ \\
\hline $\begin{array}{l}\text { Marchesoni } \\
\text { [22] }\end{array}$ & 2002 & $\mathrm{RCT}$ & $3 \mathrm{mg} / \mathrm{Kg} /$ day & 24 months & 57 & $\begin{array}{l}\text { CyA + MTX, then CyA } \\
\text { CyA + MTX, then MTX }\end{array}$ & $\begin{array}{l}\text { MTX more effective than CyA in } \\
\text { control disease activity }\end{array}$ \\
\hline $\begin{array}{l}\text { Gerards } \\
{[27]}\end{array}$ & 2002 & $\mathrm{RCT}$ & $2 \mathrm{mg} / \mathrm{kg} /$ day & 48 weeks & 120 & $\begin{array}{c}\text { CyA } \\
\text { CyA + MTX }\end{array}$ & $\begin{array}{c}\text { CyA + MTX more effective than } \\
\text { CyA in improving disease } \\
\text { activity and slowing radiologic } \\
\text { progression }\end{array}$ \\
\hline $\begin{array}{l}\text { Kvien } \\
{[15]}\end{array}$ & 2002 & $\mathrm{RCT}$ & $5 \mathrm{mg} / \mathrm{kg} /$ day & 36 months & 278 & $\begin{array}{l}\text { CyA } \\
\text { Gold }\end{array}$ & $\begin{array}{c}\text { CyA and gold equally effective in } \\
\text { preventing radiological } \\
\text { progression }\end{array}$ \\
\hline $\begin{array}{l}\text { Temekonidis } \\
\text { [39] }\end{array}$ & 2002 & CS & $2 \mathrm{mg} / \mathrm{kg} /$ day & 12 months & 18 & CyA + IFX + PDN & $\begin{array}{l}\text { Clinical improvement with the } \\
\text { addition of CyA to IFX }\end{array}$ \\
\hline $\begin{array}{l}\text { Marchesoni } \\
\text { [33] }\end{array}$ & 2003 & RCT & $3 \mathrm{mg} / \mathrm{kg} /$ day & 12 months & 61 & $\begin{array}{c}\text { CyA + MTX } \\
\text { MTX }\end{array}$ & $\begin{array}{c}\text { CyA + MTX more effective than } \\
\text { MTX in improving disease } \\
\text { activity and slowing radiologic } \\
\text { progression }\end{array}$ \\
\hline $\begin{array}{l}\text { Miranda } \\
{[24]}\end{array}$ & 2004 & RCT & $\begin{array}{c}2.5-5 \\
\mathrm{mg} / \mathrm{kg} / \text { day }\end{array}$ & 12 months & 149 & $\begin{array}{c}\text { CyA + chloroquine } \\
\text { CyA + placebo }\end{array}$ & $\begin{array}{c}\mathrm{CyA}+\text { chloroquine as effective as } \\
\text { CyA }+ \text { chloroquine }\end{array}$ \\
\hline
\end{tabular}




\begin{tabular}{|c|c|c|c|c|c|c|c|}
\hline $\begin{array}{l}\text { Sarzi Puttini } \\
\text { [23] }\end{array}$ & 2005 & RCT & $3 \mathrm{mg} / \mathrm{kg} /$ day & 12 months & 105 & $\begin{array}{c}\text { CyA } \\
\text { CyA + MTX } \\
\text { CyA + HCQ }\end{array}$ & $\begin{array}{c}\text { CyA + MTX more effective than } \\
\text { CyA and CyA + HCQ in } \\
\text { improving disease activity and } \\
\text { slow radiologic progression }\end{array}$ \\
\hline $\begin{array}{l}\text { Karanikolas } \\
{[31]}\end{array}$ & 2006 & RCT & $\begin{array}{c}2.5-4.6 \\
\mathrm{mg} / \mathrm{kg} / \text { day }\end{array}$ & 12 months & 106 & $\begin{array}{c}\text { CyA + LEF } \\
\text { CyA } \\
\text { LEF }\end{array}$ & $\begin{array}{c}\text { CyA + LEF more effective than } \\
\text { monotherapy }\end{array}$ \\
\hline $\begin{array}{l}\text { Sidiropoulos } \\
{[38]}\end{array}$ & 2006 & $\mathrm{CS}$ & $\begin{array}{c}2.5-3 \\
\mathrm{mg} / \mathrm{kg} / \mathrm{day}\end{array}$ & 24 weeks & 19 & $\mathrm{CyA}+\mathrm{MTX}+\mathrm{IFX}$ & $\begin{array}{c}\text { Modest response with CyA + IFX } \\
+ \text { MTX }\end{array}$ \\
\hline $\begin{array}{l}\text { Choy } \\
{[34]}\end{array}$ & 2008 & RCT & $3 \mathrm{mg} / \mathrm{kg} /$ day & 24 months & 467 & $\begin{array}{c}\text { CyA + MTX } \\
\text { MTX + steroids } \\
\text { CyA + MTX + steroids }\end{array}$ & $\begin{array}{l}\text { CyA + MTX + steroids more } \\
\text { effective than CyA + MTX and } \\
\text { MTX + steroids in slowing } \\
\text { radiologic progression }\end{array}$ \\
\hline $\begin{array}{l}\text { Bejarano } \\
{[209]}\end{array}$ & 2008 & RCT & $1.5 \mathrm{mg} / \mathrm{Kg} / \mathrm{day}$ & 7 years & 82 & $\begin{array}{c}\text { CyA + MTX + i.a. steroids } \\
\text { SSZ }\end{array}$ & $\begin{array}{c}\text { Better outcome with CyA + MTX } \\
+ \text { i.a. steroids than with SSZ }\end{array}$ \\
\hline $\begin{array}{l}\text { Migliore } 2010 \\
{[40]}\end{array}$ & 2010 & $\mathrm{CS}$ & $2-3 \mathrm{mg} / \mathrm{kg} /$ day & 6 months & 23 & $\mathrm{IFX}+\mathrm{CyA}+\mathrm{MTX}$ & $\begin{array}{c}\text { CyA + MTX effective in } \\
\text { maintaining disease remission } \\
\text { obtained with IFX }\end{array}$ \\
\hline $\begin{array}{l}\text { Hetland } \\
{[35]}\end{array}$ & 2010 & RCT & $4 \mathrm{mg} / \mathrm{Kg} /$ day & 5 years & 139 & $\begin{array}{c}\mathrm{CyA}+\mathrm{MTX}+\mathrm{HCQ} \\
\mathrm{MTX}+\mathrm{HCQ}+\text { placebo }\end{array}$ & $\begin{array}{c}\text { No clinical benefit from CyA as } \\
\text { compared to placebo }\end{array}$ \\
\hline $\begin{array}{l}\text { Bakker } \\
{[20]}\end{array}$ & 2010 & $\mathrm{CC}$ & 4 mg/Kg/day & 3 months & 151 & $\begin{array}{l}\text { CyA + oral MTX } \\
\text { s.c. MTX }\end{array}$ & $\begin{array}{c}\text { s.c. MTX better than CyA + oral } \\
\text { MTX in controlling disease } \\
\text { activity }\end{array}$ \\
\hline $\begin{array}{l}\text { Picchianti Diamanti } \\
\text { [41] }\end{array}$ & 2012 & $\mathrm{PC}$ & $\begin{array}{c}2-3 \\
\mathrm{mg} / \mathrm{Kg} / \text { day }\end{array}$ & 12 months & 7 & CyA + MTX & $\begin{array}{l}\text { CyA + MTX effective in } \\
\text { maintaining clinical remission } \\
\text { after ETN suspension }\end{array}$ \\
\hline
\end{tabular}

CyA: cyclosporine A; MTX: methotrexate; AZA: azathioprine; SSZ: sulphasalazine; HCQ: hydroxychloroquine; DMARD: disease-modifying antirheumatic drug; PDN: prednisone; IFX: infliximab; ETN: etanercept; CS: case-series; CC: case-control; RCT: randomized controlled trial; CRP: C reactive protein; PI: proximal inter-phalangeal joints; i.a.: intra-articular; s.c.: subcutaneous. 
Table 2. Clinical studies on cyclosporine $A$ in psoriatic arthritis.

\begin{tabular}{|c|c|c|c|c|c|c|c|}
\hline Author [Ref] & Year & $\begin{array}{l}\text { Study } \\
\text { design }\end{array}$ & CyA dose & Follow-up & $\begin{array}{c}\mathbf{N} \text { of } \\
\text { patients }\end{array}$ & Treatment groups & Outcome \\
\hline $\begin{array}{l}\text { Gupta } \\
{[47]}\end{array}$ & 1989 & $\mathrm{CC}$ & $6 \mathrm{mg} / \mathrm{Kg} /$ day & 8 weeks & 6 & CyA & $\begin{array}{l}\text { CyA effective in controlling joint and } \\
\text { skin disease }\end{array}$ \\
\hline $\begin{array}{l}\text { Steinsson } \\
{[48]}\end{array}$ & 1990 & CS & $3.5 \mathrm{mg} / \mathrm{Kg} /$ day & 6 months & 8 & CyA & $\begin{array}{l}\text { CyA effective in controlling joint and } \\
\text { skin disease }\end{array}$ \\
\hline $\begin{array}{l}\text { Salvarani } \\
\text { [49] }\end{array}$ & 1992 & $\mathrm{CS}$ & $3 \mathrm{mg} / \mathrm{Kg} /$ day & 6 months & 12 & CyA & $\begin{array}{l}\text { CyA effective in controlling joint and } \\
\text { skin disease }\end{array}$ \\
\hline $\begin{array}{l}\text { Spadaro } \\
{[51]}\end{array}$ & 1995 & $\mathrm{RCT}$ & $3-5 \mathrm{mg} / \mathrm{Kg} /$ day & 12 months & 35 & $\begin{array}{l}\text { CyA } \\
\text { MTX }\end{array}$ & $\begin{array}{c}\text { CyA as effective as MTX in disease } \\
\text { control }\end{array}$ \\
\hline $\begin{array}{l}\text { Mahrle } \\
{[50]}\end{array}$ & 1996 & $\mathrm{CS}$ & $2.7 \mathrm{mg} / \mathrm{Kg} /$ day & 6 months & 55 & CyA & $\begin{array}{l}\text { CyA effective in controlling joint and } \\
\text { skin disease }\end{array}$ \\
\hline $\begin{array}{l}\text { Macchioni } \\
{[57]}\end{array}$ & 1998 & $\mathrm{PC}$ & $3 \mathrm{mg} / \mathrm{Kg} /$ day & 24 months & 24 & CyA & $\begin{array}{l}\text { CyA effective in controlling radiological } \\
\text { disease progression in } 60 \% \text { of patients }\end{array}$ \\
\hline $\begin{array}{l}\text { Salvarani } \\
{[52]}\end{array}$ & 2001 & RCT & $3 \mathrm{mg} / \mathrm{Kg} /$ day & 24 weeks & 99 & $\begin{array}{c}\text { CyA } \\
\text { SSZ } \\
\text { Symptomatic therapy } \\
\end{array}$ & $\begin{array}{l}\text { CyA more effective than SSZ and } \\
\text { symptomatic therapy in disease control }\end{array}$ \\
\hline $\begin{array}{l}\text { Sarzi-Puttini } \\
\text { [56] }\end{array}$ & 2002 & $\mathrm{PC}$ & $3 \mathrm{mg} / \mathrm{Kg} /$ day & 24 months & 60 & CyA & CyA effective in disease control \\
\hline $\begin{array}{l}\text { D'Angelo } \\
\text { [54] }\end{array}$ & 2010 & $\mathrm{PC}$ & $3 \mathrm{mg} / \mathrm{Kg} /$ day & 24 week & 103 & $\mathrm{CyA}+\mathrm{ETN}$ & CyA + ETN effective in disease control \\
\hline $\begin{array}{l}\text { Atzeni } \\
{[55]}\end{array}$ & 2011 & $\mathrm{RCT}$ & $3 \mathrm{mg} / \mathrm{Kg} /$ day & 6 months & 41 & $\begin{array}{l}\mathrm{CyA}+\mathrm{ETN} \\
\mathrm{MTX}+\mathrm{ETN}\end{array}$ & $\begin{array}{c}\text { CyA + ETN as effective as MTX + ETN } \\
\text { in controlling joint disease but more } \\
\text { effective in controlling skin disease }\end{array}$ \\
\hline
\end{tabular}

CyA: cyclosporine A; MTX: methotrexate; SSZ: sulphasalazine; ETN: etanercept; CS: case-series; CC: case-control; RCT: randomized controlled trial; PC: prospective cohort. 
Table 3. Studies on cyclosporine A in systemic lupus erythematosus.

\begin{tabular}{|c|c|c|c|c|c|c|c|c|}
\hline Author [Ref] & Year & $\begin{array}{l}\text { Study } \\
\text { design }\end{array}$ & CyA dose & $\begin{array}{c}\text { Clinical } \\
\text { manifestations }\end{array}$ & Follow-up & $\begin{array}{c}\mathrm{N} \text { of } \\
\text { patien } \\
\text { ts }\end{array}$ & $\begin{array}{l}\text { Treatment } \\
\text { group }(s)\end{array}$ & Outcome \\
\hline $\begin{array}{l}\text { Isenberg } \\
{[61]}\end{array}$ & 1981 & CS & $\begin{array}{c}10 \\
\mathrm{mg} / \mathrm{Kg} / \text { day }\end{array}$ & Disease activity & 7 weeks & 5 & CyA + steroids & $\begin{array}{l}\text { CyA effective in the } \\
\text { management of arthralgias in } \\
\text { two patients, no improvement in } \\
\text { three patients }\end{array}$ \\
\hline $\begin{array}{l}\text { Feutren } \\
{[62]}\end{array}$ & 1987 & CS & $\begin{array}{c}5 \\
\mathrm{mg} / \mathrm{Kg} / \mathrm{day}\end{array}$ & Disease activity & 12 months & 13 & CyA & $\begin{array}{l}\text { CyA effective in reducing } \\
\text { disease activity in } 62 \% \text { of } \\
\text { patients }\end{array}$ \\
\hline $\begin{array}{l}\text { Heule } \\
{[103]}\end{array}$ & 1986 & $\mathrm{CR}$ & $\begin{array}{c}5.3 \\
\mathrm{mg} / \mathrm{Kg} / \text { day }\end{array}$ & Discoid LE & 10 weeks & 1 & CyA + steroids & $\begin{array}{l}\text { CyA + steroids not effective in } \\
\text { the management of discoid LE }\end{array}$ \\
\hline $\begin{array}{l}\text { Miescher } \\
\text { [63] }\end{array}$ & 1988 & CS & $\begin{array}{c}5 \\
\mathrm{mg} / \mathrm{Kg} / \text { day }\end{array}$ & $\begin{array}{c}\text { Disease activity } \\
\text { Diffuse proliferative } \\
\text { glomerulonephritis }\end{array}$ & $\begin{array}{c}27.1 \\
\text { months }\end{array}$ & 14 & CyA + steroids & $\begin{array}{l}\text { CyA effective in reducing } \\
\text { disease activity and improving } \\
\text { kidney function }\end{array}$ \\
\hline $\begin{array}{l}\text { Favre } \\
{[73]}\end{array}$ & 1989 & $\mathrm{PC}$ & $\begin{array}{c}5 \\
\mathrm{mg} / \mathrm{Kg} / \text { day }\end{array}$ & $\begin{array}{l}\text { Unresponsive lupus } \\
\text { nephritis }\end{array}$ & 52 months & 26 & CyA + steroids & $\begin{array}{l}\text { CyA effective in reducing } \\
\text { disease activity and improving } \\
\text { renal function as well as } \\
\text { proteinuria in } 90 \% \text { of patients }\end{array}$ \\
\hline $\begin{array}{l}\text { Balletta } \\
{[81]}\end{array}$ & 1992 & $\mathrm{CC}$ & $\begin{array}{c}1.5 \\
\mathrm{mg} / \mathrm{Kg} / \text { day }\end{array}$ & Active lupus nephritis & 12 months & 10 & $\begin{array}{c}\text { Steroids } \\
\text { CyA + steroids }\end{array}$ & $\begin{array}{l}\text { CyA + steroids more effective } \\
\text { than steroids alone in the } \\
\text { management of active lupus } \\
\text { nephritis }\end{array}$ \\
\hline $\begin{array}{l}\text { Hussein } \\
{[74]}\end{array}$ & 1993 & CS & $\begin{array}{c}2.24-4.2 \\
\mathrm{mg} / \mathrm{Kg} / \text { day }\end{array}$ & Lupus nephritis & 35 months & 5 & CyA + steroids & $\begin{array}{l}\text { Systemic and renal flares in } 3 \text { of } \\
5 \text { patients }\end{array}$ \\
\hline $\begin{array}{l}\text { Radhakrishnan } \\
\text { [86] }\end{array}$ & 1994 & $\mathrm{CC}$ & $\begin{array}{c}4-5 \\
\mathrm{mg} / \mathrm{Kg} / \mathrm{day}\end{array}$ & $\begin{array}{c}\text { Membranous lupus } \\
\text { nephritis }\end{array}$ & 43 months & 10 & CyA + steroids & $\begin{array}{l}\text { CyA + steroids effective in the } \\
\text { management of nephritic } \\
\text { syndrome in patients with } \\
\text { membranous lupus nephropathy }\end{array}$ \\
\hline
\end{tabular}




\begin{tabular}{|c|c|c|c|c|c|c|c|c|}
\hline $\begin{array}{l}\text { Miescher } \\
\text { [67] }\end{array}$ & 1994 & $\mathrm{CS}$ & $\begin{array}{c}5 \\
\mathrm{mg} / \mathrm{Kg} / \text { day }\end{array}$ & Disease activity & 7.1 years & 73 & $\begin{array}{l}\text { CyA + steroids } \\
\text { +/- MTX +/- } \\
\text { CTX }\end{array}$ & $\begin{array}{l}\text { CyA (+/- MTX +/- CTX) } \\
\text { effective as steroid-sparing agent }\end{array}$ \\
\hline $\begin{array}{l}\text { Yell } \\
{[104]}\end{array}$ & 1994 & $\mathrm{CR}$ & $\begin{array}{c}3.8-4 \\
\mathrm{mg} / \mathrm{Kg} / \text { day }\end{array}$ & Discoid LE & 24 weeks & 2 & CyA & $\begin{array}{l}\text { CyA effective in the } \\
\text { management of discoid LE }\end{array}$ \\
\hline $\begin{array}{l}\text { Tokuda } \\
{[64]}\end{array}$ & 1994 & $\mathrm{CS}$ & $\begin{array}{c}3 \\
\mathrm{mg} / \mathrm{Kg} / \text { day }\end{array}$ & SLE & 20 weeks & 10 & CyA & $\begin{array}{l}\text { CyA effective in reducing } \\
\text { disease activity and anti-dsDNA } \\
\text { antibody titers }\end{array}$ \\
\hline $\begin{array}{l}\text { Grabbe } \\
{[101]}\end{array}$ & 1995 & $\mathrm{CR}$ & $\begin{array}{c}2.5 \\
\mathrm{mg} / \mathrm{Kg} / \text { day }\end{array}$ & Subacute LE & 12 weeks & 1 & CyA & $\begin{array}{l}\text { CyA effective in the } \\
\text { management of subacute LE }\end{array}$ \\
\hline $\begin{array}{l}\text { Manger } \\
{[75]}\end{array}$ & 1996 & $\mathrm{CS}$ & $\begin{array}{c}3-5 \\
\mathrm{mg} / \mathrm{Kg} / \text { day }\end{array}$ & SLE & 64 months & 16 & CyA & $\begin{array}{l}\text { CyA effective in controlling } \\
\text { lupus manifestations }\end{array}$ \\
\hline $\begin{array}{l}\text { Caccavo } \\
{[65]}\end{array}$ & 1997 & $\mathrm{CS}$ & $\begin{array}{c}2.5-3.5 \\
\mathrm{mg} / \mathrm{Kg} / \text { day }\end{array}$ & SLE & 24 months & 30 & CyA & $\begin{array}{l}\text { CyA effective in controlling } \\
\text { lupus manifestations }\end{array}$ \\
\hline $\begin{array}{l}\text { Sugiyama } \\
{[210]}\end{array}$ & 1998 & $\mathrm{CR}$ & $\begin{array}{c}1 \\
\mathrm{mg} / \mathrm{Kg} / \text { day }\end{array}$ & Thrombocytopenia & NR & 2 & CyA + steroids & $\begin{array}{llr}\text { CyA effective in the } \\
\text { management } \\
\text { thrombocytopenia }\end{array}$ \\
\hline $\begin{array}{l}\text { Dostal } \\
{[66]}\end{array}$ & 1998 & $\mathrm{CS}$ & $\begin{array}{c}5 \\
\mathrm{mg} / \mathrm{Kg} / \text { day }\end{array}$ & $\begin{array}{l}\text { Disease activity } \\
\text { Lupus nephropathy }\end{array}$ & 12 months & 11 & CyA + steroids & $\begin{array}{l}\text { CyA effective in reducing } \\
\text { SLEDAI score, ANA and anti- } \\
\text { dsDNA titres, renal histological } \\
\text { disease activity and proteinuria }\end{array}$ \\
\hline $\begin{array}{l}\text { Tam } \\
{[76]}\end{array}$ & 1998 & $\mathrm{PC}$ & $\begin{array}{c}5 \\
\mathrm{mg} / \mathrm{Kg} / \mathrm{day}\end{array}$ & Type IV lupus nephritis & 12 months & 17 & CyA + steroids & $\begin{array}{l}\text { CyA + steroids effective in the } \\
\text { long-term management of type } \\
\text { IV lupus nephritis }\end{array}$ \\
\hline $\begin{array}{l}\text { Saeki } \\
{[102]}\end{array}$ & 2000 & $\mathrm{CR}$ & $\begin{array}{c}4 \\
\mathrm{mg} / \mathrm{Kg} / \mathrm{day} \\
\end{array}$ & Lupus profundus & 3 years & 1 & CyA + steroids & $\begin{array}{l}\text { CyA effective in the treatment of } \\
\text { lupus profundus }\end{array}$ \\
\hline $\begin{array}{l}\text { Bambauer } \\
{[211]}\end{array}$ & 2000 & $\mathrm{CS}$ & $\begin{array}{c}1-2 \\
\mathrm{mg} / \mathrm{Kg} / \text { day }\end{array}$ & SLE & 5 years & 28 & $\begin{array}{c}\mathrm{CyA}+\mathrm{PE}+ \\
\text { steroids }+ \\
\mathrm{AZA}+/-\mathrm{CTX}\end{array}$ & $\begin{array}{l}\text { CyA effective in controlling } \\
\text { lupus manifestations when } \\
\text { associated with PE + steroids + } \\
\text { AZA +/- CTX }\end{array}$ \\
\hline $\begin{array}{l}\text { Morton } \\
{[69]}\end{array}$ & 2000 & $\mathrm{CS}$ & $\begin{array}{c}4 \\
\mathrm{mg} / \mathrm{Kg} / \text { day }\end{array}$ & SLE & 3 years & 43 & CyA & $\begin{array}{l}\text { CyA effective in the } \\
\text { management } \\
\text { thrombocytopenia but not of } \\
\text { other lupus manifestations }\end{array}$ \\
\hline
\end{tabular}




\begin{tabular}{|c|c|c|c|c|c|c|c|c|}
\hline & & & & & & & & $\begin{array}{l}\text { (arthralgia, arthritis, myalgia, } \\
\text { fatigue) }\end{array}$ \\
\hline $\begin{array}{l}\text { Dammacco } \\
{[68]}\end{array}$ & 2000 & RCT & $\begin{array}{c}5 \\
\mathrm{mg} / \mathrm{Kg} / \text { day }\end{array}$ & SLE & 24 months & 18 & $\begin{array}{c}\text { CyA + PDN } \\
\text { PDN }\end{array}$ & $\begin{array}{l}\text { CyA effective as steroid-sparing } \\
\text { agent in SLE }\end{array}$ \\
\hline $\begin{array}{l}\text { Hallegua } \\
\text { [87] }\end{array}$ & 2000 & $\mathrm{CS}$ & $\begin{array}{c}3.8 \\
\mathrm{mg} / \mathrm{Kg} / \mathrm{day}\end{array}$ & $\begin{array}{l}\text { Membranous nephritis } \\
\text { Disease activity }\end{array}$ & 12 months & 10 & CyA & $\begin{array}{l}\text { CyA effective in reducing } \\
\text { proteinuria and and increasing } \\
\text { serum albumina, no significant } \\
\text { changes in SLEDAI score }\end{array}$ \\
\hline $\begin{array}{l}\text { Duarte-Salazar } \\
\text { [98] }\end{array}$ & 2000 & $\mathrm{CR}$ & $\begin{array}{c}3.5 \\
\mathrm{mg} / \mathrm{Kg} / \text { day } \\
\end{array}$ & Pure red cell aplasia & 6 months & 1 & CyA + steroids & $\begin{array}{l}\text { CyA effective in the treatment of } \\
\text { pure red cell aplasia }\end{array}$ \\
\hline $\begin{array}{l}\text { Tam } \\
{[88]}\end{array}$ & 2001 & $\mathrm{CS}$ & $\begin{array}{c}5 \\
\mathrm{mg} / \mathrm{Kg} / \text { day }\end{array}$ & Type IV lupus nephritis & 48 months & 17 & CyA + steroids & $\begin{array}{l}\text { CyA + steroids effective in lupus } \\
\text { nephritis, with reduction of } \\
\text { proteinuria, increase of serum } \\
\text { albumin and histological } \\
\text { improvement }\end{array}$ \\
\hline $\begin{array}{l}\text { Atzeni } \\
{[97]}\end{array}$ & 2003 & $\mathrm{CR}$ & $200 \mathrm{mg} / \mathrm{day}$ & Pure red cell aplasia & 2 years & 1 & CyA + steroids & $\begin{array}{l}\text { CyA effective in the treatment of } \\
\text { pure red cell aplasia }\end{array}$ \\
\hline $\begin{array}{l}\mathrm{Hu} \\
{[89]}\end{array}$ & 2003 & $\mathrm{CS}$ & $\begin{array}{c}5 \\
\mathrm{mg} / \mathrm{Kg} / \mathrm{day}\end{array}$ & $\begin{array}{l}\text { Membranous } \\
\text { nephropathy }\end{array}$ & 36 months & 24 & CyA & $\begin{array}{l}\text { CyA effective in inducing } \\
\text { complete remission in } 52.2 \% \text { of } \\
\text { patients, partial remission in } \\
43.3 \%\end{array}$ \\
\hline $\begin{array}{l}\text { Singh } \\
{[96]}\end{array}$ & 2004 & $\mathrm{CR}$ & $300 \mathrm{mg} / \mathrm{day}$ & Aplastic anaemia & 4 months & 1 & $\begin{array}{c}\text { CyA + steroids } \\
+ \text { CTX }\end{array}$ & $\begin{array}{l}\text { CyA effective in the treatment of } \\
\text { aplastic anemia }\end{array}$ \\
\hline $\begin{array}{l}\text { Arcasoy } \\
{[99]}\end{array}$ & 2005 & $\mathrm{CR}$ & $\begin{array}{c}5 \\
\mathrm{mg} / \mathrm{Kg} / \text { day }\end{array}$ & Pure red cell aplasia & 3 months & 1 & $\mathrm{CyA}+\mathrm{MMF}$ & $\begin{array}{l}\text { CyA effective in the treatment of } \\
\text { pure red cell aplasia }\end{array}$ \\
\hline $\begin{array}{l}\text { Wang } \\
{[95]}\end{array}$ & 2005 & $\mathrm{CR}$ & $\begin{array}{c}3 \\
\mathrm{mg} / \mathrm{Kg} / \text { day }\end{array}$ & $\begin{array}{c}\text { Refractory haemolytic } \\
\text { anaemia }\end{array}$ & 2 months & 1 & CyA + steroids & $\begin{array}{l}\text { CyA effective in the treatment of } \\
\text { refractory haemolytic anaemia }\end{array}$ \\
\hline $\begin{array}{l}\text { Moroni } \\
{[82]}\end{array}$ & 2006 & RCT & $\begin{array}{c}4 \\
\mathrm{mg} / \mathrm{Kg} / \text { day }\end{array}$ & $\begin{array}{c}\text { Proliferative lupus } \\
\text { nephritis }\end{array}$ & 4 months & 69 & $\begin{array}{l}\text { CyA } \\
\text { AZA }\end{array}$ & $\begin{array}{l}\text { CyA and AZA equally effective } \\
\text { as maintenance treatment of } \\
\text { proliferative lupus nephritis }\end{array}$ \\
\hline $\begin{array}{l}\text { Quartuccio } \\
\text { [93] }\end{array}$ & 2006 & $\mathrm{CS}$ & $\begin{array}{c}3-5 \\
\mathrm{mg} / \mathrm{Kg} / \mathrm{day}\end{array}$ & Thrombocytopenia & $\begin{array}{c}23.5 \\
\text { months }\end{array}$ & 6 & CyA + steroids & $\begin{array}{l}\text { CyA effective in the } \\
\text { management } \\
\text { thrombocytopenia }\end{array}$ \\
\hline Ogawa & 2007 & $\mathrm{CS}$ & 2.5 & Refractory lupus & 30 weeks & 9 & CyA + steroids & CyA + steroids effective in \\
\hline
\end{tabular}




\begin{tabular}{|c|c|c|c|c|c|c|c|c|}
\hline [78] & & & $\mathrm{mg} / \mathrm{Kg} /$ day & nephritis & & & & $\begin{array}{l}\text { refractory lupus nephritis, with } \\
\text { reduction of proteinuria }\end{array}$ \\
\hline $\begin{array}{l}\text { Rihova } \\
{[77]}\end{array}$ & 2007 & $\mathrm{CS}$ & $\begin{array}{c}5 \\
\mathrm{mg} / \mathrm{Kg} / \mathrm{day}\end{array}$ & Lupus nephritis & $\begin{array}{c}85.6 \\
\text { months }\end{array}$ & 31 & CyA & $\begin{array}{l}\text { CyA effective in reducing } \\
\text { proteinuria and stabilizing renal } \\
\text { function }\end{array}$ \\
\hline $\begin{array}{l}\text { Austin } \\
{[90]}\end{array}$ & 2009 & RCT & NR & $\begin{array}{l}\text { Membranous lupus } \\
\text { nephritis }\end{array}$ & 12 months & 42 & $\begin{array}{l}\text { CyA } \\
\text { CTX } \\
\text { PDN }\end{array}$ & $\begin{array}{l}\text { CyA more effective than PDN } \\
\text { but less effective than CTX in } \\
\text { the maintenance treatment of } \\
\text { proliferative lupus nephritis }\end{array}$ \\
\hline $\begin{array}{l}\text { Zavada } \\
{[83]}\end{array}$ & 2010 & RCT & $\begin{array}{c}4-5 \\
\mathrm{mg} / \mathrm{Kg} / \text { day }\end{array}$ & $\begin{array}{l}\text { Proliferative lupus } \\
\text { nephritis }\end{array}$ & 18 months & 40 & $\begin{array}{l}\text { CyA } \\
\text { CTX }\end{array}$ & $\begin{array}{l}\text { CyA as effective as CTX in the } \\
\text { induction and maintenance } \\
\text { treatment in patients with } \\
\text { proliferative lupus nephritis }\end{array}$ \\
\hline $\begin{array}{l}\text { Ogawa } \\
{[79]}\end{array}$ & 2010 & $\mathrm{CS}$ & $\begin{array}{c}2.5 \\
\mathrm{mg} / \mathrm{Kg} / \text { day }\end{array}$ & Disease activity & $\begin{array}{c}21.5 \\
\text { months }\end{array}$ & 55 & CyA & $\begin{array}{l}\text { CyA effective in reducing } \\
\text { SLEDAI and flare rate }\end{array}$ \\
\hline $\begin{array}{l}\text { Kamijo } \\
{[80]}\end{array}$ & 2011 & $\mathrm{CS}$ & $\begin{array}{c}2.5 \\
\mathrm{mg} / \mathrm{Kg} / \text { day }\end{array}$ & $\begin{array}{c}\text { Proliferative lupus } \\
\text { nephritis }\end{array}$ & 2 years & 11 & CyA & $\begin{array}{l}\text { CyA effective as treatment of } \\
\text { proliferative lupus nephritis }\end{array}$ \\
\hline $\begin{array}{l}\text { Zavada } \\
{[84]}\end{array}$ & 2014 & RCT & $\begin{array}{c}4.5 \\
\mathrm{mg} / \mathrm{Kg} / \mathrm{day}\end{array}$ & $\begin{array}{l}\text { Proliferative lupus } \\
\text { nephritis }\end{array}$ & 7.7 years & 38 & $\begin{array}{l}\text { CyA } \\
\text { CTX }\end{array}$ & $\begin{array}{l}\text { CyA and CTX equally effective } \\
\text { at long-term as treatment of } \\
\text { proliferative lupus nephritis }\end{array}$ \\
\hline $\begin{array}{l}\text { Takahashi } \\
\text { [100] }\end{array}$ & 2015 & $\mathrm{CS}$ & NR & $\begin{array}{c}\text { Lupus } \\
\text { haemophagocytic } \\
\text { syndrome }\end{array}$ & NR & 3 & CyA + steroids & $\begin{array}{l}\text { CyA effective in the treatment of } \\
\text { lupus } \\
\text { syndrome }\end{array}$ \\
\hline
\end{tabular}

CyA: cyclosporine A; CTX: cyclophosphamide; PDN: prednisone; MTX: methotrexate; AZA: azathioprine; MMF: mycophenolate mofetil; PE: plasma exchange: SLE: systemic lupus erythematosus; LE: lupus erythematosus; SLEDAI: systemic lupus erythematosus disease activity index;

NR: not reported; ANA: anti-nuclear antibodies; anti-dsDNA: anti-double stranded DNA antibodies. 
Table 4. Studies on cyclosporine A in systemic sclerosis.

\begin{tabular}{|c|c|c|c|c|c|c|c|}
\hline Author [Ref] & Year & $\begin{array}{l}\text { Study } \\
\text { design }\end{array}$ & CyA dose & Follow-up & $\mathrm{N}$ of patients & Treatment groups & Outcome \\
\hline $\begin{array}{l}\text { Zachariae } \\
\text { [109] }\end{array}$ & 1990 & PC & $\begin{array}{c}7.5 \\
\mathrm{mg} / \mathrm{Kg} / \text { day }\end{array}$ & 16 months & 10 & CyA & $\begin{array}{l}\text { CyA effective in reducing skin } \\
\text { thickening in } 20 \% \text { of patients }\end{array}$ \\
\hline $\begin{array}{l}\text { Clements } \\
{[108]}\end{array}$ & 1993 & $\mathrm{PC}$ & $\begin{array}{c}5 \\
\mathrm{mg} / \mathrm{Kg} / \mathrm{day}\end{array}$ & 48 week & 10 & CyA & $\begin{array}{l}\text { CyA effective in reducing skin } \\
\text { thickening but not in controlling } \\
\text { lung and cardiac involvement }\end{array}$ \\
\hline $\begin{array}{l}\text { Denton } \\
{[115]}\end{array}$ & 1994 & $\mathrm{RCT}$ & $\begin{array}{c}2.5 \\
\mathrm{mg} / \mathrm{Kg} / \text { day }\end{array}$ & 1 year & 20 & $\begin{array}{l}\text { CyA + iloprost } \\
\text { Iloprost + placebo }\end{array}$ & $\begin{array}{l}\text { CyA effective in reducing skin } \\
\text { thickening and controlling } \\
\text { microvascular and oesophageal } \\
\text { involvement }\end{array}$ \\
\hline $\begin{array}{l}\text { Filaci } \\
{[111]}\end{array}$ & 1999 & $\mathrm{CR}$ & $\begin{array}{c}3-4 \\
\mathrm{mg} / \mathrm{Kg} / \text { day }\end{array}$ & - & 3 & CyA & SRC in all cases \\
\hline $\begin{array}{l}\text { Morton } \\
{[212]}\end{array}$ & 2000 & CS & $\begin{array}{c}3.4 \\
\mathrm{mg} / \mathrm{Kg} / \text { day }\end{array}$ & - & 16 & CyA & $\begin{array}{l}\text { CyA effective in reducing skin } \\
\text { thickening in half of patients }\end{array}$ \\
\hline $\begin{array}{l}\text { Roch } \\
{[110]}\end{array}$ & 2004 & CR & $\begin{array}{c}2 \\
\mathrm{mg} / \mathrm{Kg} / \mathrm{day}\end{array}$ & 5 years & 1 & CyA & $\begin{array}{l}\text { CyA effective in reducing skin } \\
\text { thickening }\end{array}$ \\
\hline $\begin{array}{l}\text { Zentilin } \\
{[112]}\end{array}$ & 2004 & CR & $\begin{array}{c}2.5 \\
\mathrm{mg} / \mathrm{Kg} / \text { day }\end{array}$ & 6 months & 2 & CyA & $\begin{array}{l}\text { CyA effective in controlling } \\
\text { oesophageal involvement }\end{array}$ \\
\hline $\begin{array}{l}\text { Ando } \\
{[113]}\end{array}$ & 2013 & $\mathrm{CR}$ & $\begin{array}{l}100-150 \\
\text { mg/day }\end{array}$ & 2-4 years & 2 & CyA & $\begin{array}{l}\text { Stabilization of lung function } \\
\text { with CyA as maintenance } \\
\text { treatment }\end{array}$ \\
\hline
\end{tabular}

CyA: cyclosporine A; SRC: scleroderma renal crisis; CS: case-series; PC: prospective cohort; CR: case-report; RCT: randomized controlled trial. 
Table 5. Studies on cyclosporine A in inflammatory myopathies.

\begin{tabular}{|c|c|c|c|c|c|c|c|}
\hline Author [Ref] & Year & $\begin{array}{l}\text { Study } \\
\text { design }\end{array}$ & CyA dose & Follow-up & $\mathbf{N}$ of patients & Treatment groups & Outcome \\
\hline $\begin{array}{l}\text { Grau } \\
{[116]}\end{array}$ & 1994 & $\mathrm{CC}$ & $\begin{array}{c}5 \\
\mathrm{mg} / \mathrm{Kg} / \mathrm{day}\end{array}$ & 24 months & $10 \mathrm{DM}$ & $\begin{array}{c}\text { CyA } \\
\text { Steroids + AZA }\end{array}$ & $\begin{array}{l}\text { CyA effective in inducing } \\
\text { remissions more quickly than } \\
\text { steroids + AZA }\end{array}$ \\
\hline Vencovsky & 2000 & $\mathrm{RCT}$ & $\begin{array}{c}3-3.5 \\
\mathrm{mg} / \mathrm{Kg} / \text { day }\end{array}$ & 6 months & $\begin{array}{l}20 \mathrm{DM} \\
16 \mathrm{PM}\end{array}$ & $\begin{array}{l}\text { CyA + steroids } \\
\text { MTX + steroids }\end{array}$ & $\begin{array}{c}\text { CyA and MTX equally effective in } \\
\text { the management of muscle } \\
\text { involvement }\end{array}$ \\
\hline $\begin{array}{l}\text { Kotani } \\
{[119]}\end{array}$ & 2005 & $\mathrm{CR}$ & NR & NR & $1 \mathrm{DM}$ & CyA + CTX + steroids & $\begin{array}{c}\mathrm{CyA}+\text { steroids not effective in the } \\
\text { management of ILD }\end{array}$ \\
\hline $\begin{array}{l}\text { Takada } \\
{[122]}\end{array}$ & 2005 & $\mathrm{RC}$ & $150 \mathrm{mg} / \mathrm{day}$ & 24 months & $53 \mathrm{DM} / \mathrm{PM}$ & $\begin{array}{l}\text { CyA + steroids } \\
\text { Steroids }\end{array}$ & $\begin{array}{c}\text { CyA + steroids more effective than } \\
\text { steroids alone in managing } \\
\text { PM/DM }\end{array}$ \\
\hline $\begin{array}{l}\text { Sakamoto } \\
{[124]}\end{array}$ & 2005 & $\mathrm{CR}$ & $\begin{array}{l}100-150 \\
\text { mg/day }\end{array}$ & 7.5 years & $1 \mathrm{DM}$ & CyA + steroids & $\begin{array}{c}\text { CyA + steroids effective in the } \\
\text { management of ILD }\end{array}$ \\
\hline $\begin{array}{l}\text { Mii } \\
{[118]}\end{array}$ & 2006 & $\mathrm{CR}$ & $\begin{array}{c}1 \\
\mathrm{mg} / \mathrm{Kg} / \text { day }\end{array}$ & 6 months & $1 \mathrm{DM}$ & $\mathrm{CyA}+$ steroids & $\begin{array}{c}\text { CyA + steroids effective in the } \\
\text { management of dysphagia }\end{array}$ \\
\hline $\begin{array}{l}\text { Terao } \\
{[121]}\end{array}$ & 2007 & CR & $125 \mathrm{mg} / \mathrm{day}$ & 4 years & $1 \mathrm{DM}$ & CyA + steroids & $\begin{array}{c}\text { CyA + steroids effective in the } \\
\text { management of } \\
\text { pneumomediastinum }\end{array}$ \\
\hline $\begin{array}{l}\text { Kotani } \\
{[120]}\end{array}$ & 2008 & $\mathrm{CS}$ & $\begin{array}{c}4 \\
\mathrm{mg} / \mathrm{Kg} / \text { day }\end{array}$ & 2 weeks & $16 \mathrm{DM}$ & $\mathrm{CyA}+$ steroids & $\begin{array}{l}\text { CyA effective as early treatment of } \\
\text { DM-ILD }\end{array}$ \\
\hline $\begin{array}{l}\text { Kim } \\
{[213]}\end{array}$ & 2009 & $\mathrm{CR}$ & $100 \mathrm{mg} /$ day & NR & $6 \mathrm{DM}$ & CyA + steroids & $\begin{array}{l}\text { CyA effective in the management } \\
\text { of } 5 \text { out of } 6 \text { patients with DM } \\
\text { complicated } \\
\text { pneumomediastinum }\end{array}$ \\
\hline $\begin{array}{l}\text { Kotani } \\
{[214]}\end{array}$ & 2011 & CS & $\begin{array}{c}4 \\
\mathrm{mg} / \mathrm{Kg} / \text { day }\end{array}$ & 12 months & $14 \mathrm{DM}$ & CyA + steroids & $\begin{array}{l}\text { CyA + steroids effective in } \\
\text { improving LFT and HRCT }\end{array}$ \\
\hline
\end{tabular}




\begin{tabular}{|l|c|c|c|c|c|c|c|}
\hline $\begin{array}{l}\text { Ingegnoli } \\
{[127]}\end{array}$ & 2012 & $\mathrm{CC}$ & $\begin{array}{c}5 \\
\mathrm{mg} / \mathrm{Kg} / \mathrm{day}\end{array}$ & 12 months & $15 \mathrm{PM}$ & $\begin{array}{l}\text { CyA + steroids } \\
\text { CTX + steroids }\end{array}$ & $\begin{array}{l}\text { No significant difference between } \\
\text { CyA and CTX groups in the } \\
\text { progression rate of ILD at HRCT }\end{array}$ \\
\hline $\begin{array}{l}\text { Suzuki } \\
{[126]}\end{array}$ & 2013 & $\mathrm{CR}$ & $150 \mathrm{mg} / \mathrm{day}$ & $\mathrm{NR}$ & $3 \mathrm{DM}$ & $\mathrm{CyA}+$ steroids + CTX & $\begin{array}{l}\text { CyA + steroids + CTX effective in } \\
\text { the management of DM-ILD }\end{array}$ \\
\hline $\begin{array}{l}\text { Cavagna } \\
{[125]}\end{array}$ & 2013 & $\mathrm{CS}$ & $\begin{array}{c}3 \\
\mathrm{mg} / \mathrm{Kg} / \mathrm{day}\end{array}$ & 96 months & $18 \mathrm{PM}$ & $\mathrm{CyA}+$ steroids & $\begin{array}{l}\text { CyA effective in the management } \\
\text { of DM-ILD }\end{array}$ \\
\hline $\begin{array}{l}\text { Labirua- } \\
\text { Iturburu } \\
{[123]}\end{array}$ & 2013 & $\mathrm{CS}$ & $\begin{array}{c}2.5-5 \\
\mathrm{mg} / \mathrm{Kg} / \mathrm{day}\end{array}$ & 24 months & $15 \mathrm{PM}$ & $\begin{array}{l}\text { CyA } \\
\text { Tacrolimus effective in the management } \\
\text { of DM-ILD }\end{array}$ \\
\hline
\end{tabular}

DM: dermatomyositis; PM: polymyositis; ILD: interstitial lung disease; CyA: cyclosporine A; MTX: methotrexate; CTX: cyclophosphamide; AZA: azathioprine; LFT: lung function test; HRCT: high resolution CT scan of the chest; CC: case cohort; RC: retrospective cohort; CS: case-series; CR: case-report; RCT: randomized controlled trial; NR: not reported. 
Table 6. Studies on cyclosporine A in systemic vasculitides.

\begin{tabular}{|c|c|c|c|c|c|c|c|c|}
\hline Author [Ref] & Year & $\begin{array}{l}\text { Study } \\
\text { design }\end{array}$ & & CyA dose & Follow-up & $\begin{array}{c}\mathrm{N} \text { of } \\
\text { patients }\end{array}$ & Treatment groups & Outcome \\
\hline $\begin{array}{l}\text { Wendling } \\
{[134]}\end{array}$ & 1985 & CR & $\begin{array}{c}\text { PMR } \\
\text { TA }\end{array}$ & $\begin{array}{c}3 \\
\mathrm{mg} / \mathrm{Kg} / \text { day }\end{array}$ & 3 months & 2 & CyA + steroids & $\begin{array}{l}\text { CyA effective in the } \\
\text { management of relapsing } \\
\text { PMR and TA }\end{array}$ \\
\hline $\begin{array}{l}\text { Gremmel } \\
{[128]}\end{array}$ & 1988 & $\mathrm{CR}$ & GPA & $\begin{array}{c}5 \\
\mathrm{mg} / \mathrm{Kg} / \mathrm{day}\end{array}$ & 27 months & 2 & CyA + steroids & $\begin{array}{l}\text { CyA effective of } \\
\text { pulmonary and renal } \\
\text { involvement }\end{array}$ \\
\hline $\begin{array}{l}\text { Harley } \\
{[130]}\end{array}$ & 1990 & $\mathrm{CR}$ & GPA & NR & 6 months & 1 & CyA & $\begin{array}{l}\text { CyA effective in the } \\
\text { management of GPA }\end{array}$ \\
\hline $\begin{array}{l}\text { Borleffs } \\
{[129]}\end{array}$ & 1990 & $\mathrm{CR}$ & GPA & $\begin{array}{c}5 \\
\mathrm{mg} / \mathrm{Kg} / \text { day }\end{array}$ & 2 years & 1 & CyA + steroids & $\begin{array}{l}\text { CyA effective in the } \\
\text { management of severe } \\
\text { GPA with renal } \\
\text { involvement }\end{array}$ \\
\hline $\begin{array}{l}\text { Fullerton } \\
{[138]}\end{array}$ & 1991 & $\mathrm{CR}$ & TA & $\begin{array}{c}4 \\
\mathrm{mg} / \mathrm{Kg} / \text { day }\end{array}$ & 3 months & 1 & CyA & $\begin{array}{l}\text { CyA effective in the } \\
\text { management of pyoderma } \\
\text { gangrenosum associated } \\
\text { with TA }\end{array}$ \\
\hline $\begin{array}{l}\text { Schaufelberger } \\
\text { [133] }\end{array}$ & 1998 & $\mathrm{RCT}$ & GCA & $\begin{array}{c}2 \\
\mathrm{mg} / \mathrm{Kg} / \mathrm{day}\end{array}$ & 6 months & 22 & $\begin{array}{c}\text { CyA + steroids } \\
\text { CyA } \\
\end{array}$ & $\begin{array}{l}\text { CyA not effective as } \\
\text { additive agent in GCA }\end{array}$ \\
\hline $\begin{array}{l}\text { McDermott } \\
{[132]}\end{array}$ & 1998 & $\mathrm{CR}$ & EGP & $\begin{array}{c}3.5 \\
\mathrm{mg} / \mathrm{Kg} / \mathrm{day}\end{array}$ & 5 months & 1 & CyA + steroids & $\begin{array}{l}\text { CyA effective in the } \\
\text { management of severe } \\
\text { EGP }\end{array}$ \\
\hline $\begin{array}{l}\text { Fearfield } \\
{[137]}\end{array}$ & 1999 & $\mathrm{CR}$ & TA & $\begin{array}{c}5 \\
\mathrm{mg} / \mathrm{Kg} / \mathrm{day}\end{array}$ & 18 months & 1 & $\begin{array}{l}\mathrm{CyA}+\text { steroids }+ \\
\text { minocycline }\end{array}$ & $\begin{array}{l}\text { CyA effective in the } \\
\text { management of pyoderma } \\
\text { gangrenosum associated } \\
\text { with TA }\end{array}$ \\
\hline $\begin{array}{l}\text { Horigome } \\
\text { [136] }\end{array}$ & 1999 & $\mathrm{CR}$ & $\mathrm{TA}$ & $\begin{array}{c}4.3 \\
\mathrm{mg} / \mathrm{Kg} / \text { day }\end{array}$ & 4 years & 1 & CyA + steroids & $\begin{array}{l}\text { CyA effective in the } \\
\text { management of TA }\end{array}$ \\
\hline $\begin{array}{l}\text { Inoue } \\
{[131]}\end{array}$ & 2000 & $\mathrm{CR}$ & GPA & $\begin{array}{c}100 \\
\mathrm{mg} / \text { day }\end{array}$ & NR & 1 & $\begin{array}{l}\text { CyA + CTX + } \\
\text { steroids }\end{array}$ & $\begin{array}{l}\text { CyA effective in the } \\
\text { management of GPA }\end{array}$ \\
\hline
\end{tabular}


PMR: polymyalgia rheumatica; GPA: granulomatosis with polyangiitis; GCA: giant cell arteritis; TA: Takayasu's arteritis; EGP: esinophilic granulomatosis with polyangiitis; CyA: cyclosporine A; CTX: cyclophosphamide; CR: case-report; RCT: randomized controlled trial; NR: not reported. 
Table 7. Studies on cyclosporine A in Behçet's disease.

\begin{tabular}{|c|c|c|c|c|c|c|c|}
\hline $\begin{array}{l}\text { Author } \\
\text { [Ref] }\end{array}$ & Year & $\begin{array}{l}\text { Study } \\
\text { design }\end{array}$ & CyA dose & $\mathrm{N}$ of patients & Follow-up & Treatment groups & Outcome \\
\hline $\begin{array}{l}\text { BenEzra } \\
{[142]}\end{array}$ & 1988 & $\mathrm{CC}$ & $\begin{array}{c}5 \\
\mathrm{mg} / \mathrm{Kg} / \text { day }\end{array}$ & 40 & 3 years & $\begin{array}{l}\text { CyA } \\
\text { Corticosteroids } \\
\text { Leukeran }\end{array}$ & $\begin{array}{l}\text { CyA more effective than } \\
\text { conventional treatment in the } \\
\text { management of ocular } \\
\text { manifestations of BD }\end{array}$ \\
\hline $\begin{array}{l}\text { Masuda } \\
\text { [143] }\end{array}$ & 1989 & $\mathrm{RCT}$ & $\begin{array}{c}10 \\
\mathrm{mg} / \mathrm{Kg} / \mathrm{day}\end{array}$ & 96 & 16 weeks & $\begin{array}{c}\text { CyA } \\
\text { Colchicine }\end{array}$ & $\begin{array}{l}\text { CyA more effective than colchicine } \\
\text { in reducing the frequency and } \\
\text { severity of ocular attacks, of oral } \\
\text { aphthous ulcer and dermal lesions }\end{array}$ \\
\hline $\begin{array}{l}\text { Ozyazgan } \\
{[141]}\end{array}$ & 1992 & $\mathrm{CC}$ & $\begin{array}{c}5 \\
\mathrm{mg} / \mathrm{Kg} / \mathrm{day}\end{array}$ & 23 & 34 months & $\begin{array}{l}\text { CyA } \\
\text { CTX }\end{array}$ & $\begin{array}{c}\text { CyA more effective than CTX in the } \\
\text { management of BD-associated } \\
\text { uveitis at } 6 \text { months }\end{array}$ \\
\hline $\begin{array}{l}\text { Avci } \\
{[148]}\end{array}$ & 1997 & $\mathrm{CS}$ & $\begin{array}{c}5 \\
\mathrm{mg} / \mathrm{Kg} / \mathrm{day}\end{array}$ & 24 & 6 months & CyA & $\begin{array}{c}\text { CyA effective on mucocutaneous } \\
\text { manifestations of BD }\end{array}$ \\
\hline $\begin{array}{l}\text { Cantini } \\
{[147]}\end{array}$ & 1999 & $\mathrm{PC}$ & $\begin{array}{c}5 \\
\mathrm{mg} / \mathrm{Kg} / \mathrm{day}\end{array}$ & 7 & 48 months & CyA & $\begin{array}{l}\text { CyA effective in the management of } \\
\text { thrombophlebitis associated with BD }\end{array}$ \\
\hline $\begin{array}{l}\text { Yamada } \\
{[144]}\end{array}$ & 2010 & $\mathrm{RCT}$ & $\begin{array}{c}3-5 \\
\mathrm{mg} / \mathrm{Kg} / \text { day }\end{array}$ & 37 & 6 months & $\begin{array}{l}\text { CyA } \\
\text { IFX }\end{array}$ & $\begin{array}{l}\text { CyA less effective than IFX in } \\
\text { reducing episodes of uveitis in BD }\end{array}$ \\
\hline
\end{tabular}

BD: Behçet's disease; CyA: cyclosporine A; CTX: cyclophosphamide; IFX: infliximab; CS: case-series; CC: case-control; RCT: randomized controlled trial; PC: prospective cohort. 
Table 8. Studies on cyclosporine A in adult onset Still's disease.

\begin{tabular}{|c|c|c|c|c|c|c|c|}
\hline Author [Ref] & Year & $\begin{array}{l}\text { Study } \\
\text { design }\end{array}$ & CyA dose & Follow-up & $\mathbf{N}$ of patients & Treatment group(s) & Outcome \\
\hline $\begin{array}{l}\text { Mori } \\
{[155]}\end{array}$ & 1993 & $\mathrm{CR}$ & NR & NR & 1 & CyA + steroids & $\begin{array}{l}\text { Improvement of Still's disease- } \\
\text { associated DIC with CyA }\end{array}$ \\
\hline $\begin{array}{l}\text { Park } \\
{[156]}\end{array}$ & 2004 & $\mathrm{CR}$ & $\begin{array}{c}2-3 \\
\mathrm{mg} / \mathrm{Kg} / \text { day } \\
\end{array}$ & 12 months & 1 & CyA + steroids + IvIg & $\begin{array}{l}\text { Improvement of Still's disease- } \\
\text { associated DIC with CyA }\end{array}$ \\
\hline $\begin{array}{l}\text { Hamidou } \\
{[157]}\end{array}$ & 2005 & $\mathrm{CR}$ & NR & NR & 1 & CyA & $\begin{array}{c}\text { Improvement of Still's disease- } \\
\text { associated haemophagocytic } \\
\text { syndrome with CyA }\end{array}$ \\
\hline $\begin{array}{l}\text { Her } \\
{[159]}\end{array}$ & 2007 & $\mathrm{CR}$ & $\begin{array}{c}300 \\
\mathrm{mg} / \mathrm{day}\end{array}$ & 48 months & 1 & CyA & $\begin{array}{l}\text { Improvement of Still's disease- } \\
\text { associated acquired } \\
\text { amegakaryocytic } \\
\text { thrombocytopenia with CyA }\end{array}$ \\
\hline $\begin{array}{l}\text { Nagashima } \\
{[160]}\end{array}$ & 2008 & $\mathrm{CR}$ & $\begin{array}{c}3 \\
\mathrm{mg} / \mathrm{Kg} / \mathrm{day}\end{array}$ & NR & 2 & CyA & $\begin{array}{c}\text { Improvement of Still's disease- } \\
\text { associated hepatic failure with } \\
\text { CyA }\end{array}$ \\
\hline $\begin{array}{l}\text { Mizrahi } \\
{[158]}\end{array}$ & 2009 & $\mathrm{CR}$ & $\begin{array}{c}3 \\
\mathrm{mg} / \mathrm{Kg} / \mathrm{day}\end{array}$ & 8 months & 1 & $\begin{array}{c}\mathrm{CyA}+\mathrm{MMF}+ \\
\text { steroids }\end{array}$ & $\begin{array}{l}\text { Improvement of Still's disease- } \\
\text { associated relapsing macrophage } \\
\text { activating syndrome with CyA }\end{array}$ \\
\hline $\begin{array}{l}\text { Mitamura } \\
{[153]}\end{array}$ & 2009 & $\mathrm{CS}$ & $\begin{array}{c}\text { Adjusted } \\
\text { dose }\end{array}$ & 12.4 months & 7 & CyA & $\begin{array}{l}\text { Induction of remission in } 6 / 7 \\
\text { patients with Still's disease }\end{array}$ \\
\hline
\end{tabular}

CyA: cyclosporine A; DIC: disseminated intravascular coagulation; NR: not reported; MMF: mycophenolate mofetil; IvIg: intra-venous Immunoglobulins; CR: case-reports; CS: case-series. 
Table 9. Studies on cyclosporine A in Sjogren's syndrome or dry eye disease.

\begin{tabular}{|c|c|c|c|c|c|c|c|}
\hline $\begin{array}{l}\text { Author } \\
\text { [Ref] }\end{array}$ & Year & $\begin{array}{l}\text { Study } \\
\text { Design }\end{array}$ & CyA dose & $\mathbf{N}$ of patients & Follow-up & Treatment groups & Outcome \\
\hline $\begin{array}{l}\text { Drosos } \\
{[170]}\end{array}$ & 1986 & $\mathrm{CS}$ & $\begin{array}{c}5 \\
\mathrm{mg} / \mathrm{Kg} / \text { day } \\
\end{array}$ & $20 \mathrm{SSj}$ & 12 months & CyA & $\begin{array}{l}\text { CyA effective on xerostomia but not } \\
\text { minor salivary gland histology }\end{array}$ \\
\hline $\begin{array}{l}\text { Drosos } \\
{[171]}\end{array}$ & 1986 & $\mathrm{CC}$ & $\begin{array}{c}5 \\
\mathrm{mg} / \mathrm{Kg} / \text { day } \\
\end{array}$ & $20 \mathrm{SSj}$ & 6 months & $\begin{array}{c}\text { CyA } \\
\text { Placebo }\end{array}$ & $\begin{array}{c}\text { CyA effective on histology but not } \\
\text { xerostomia }\end{array}$ \\
\hline $\begin{array}{l}\text { Stevenson } \\
{[165]}\end{array}$ & 2000 & $\mathrm{RCT}$ & $\begin{array}{c}\text { Topical } \\
0.05 \% \text { - } \\
0.1 \% \text { - } \\
0.2 \% \text { - } \\
0.4 \%\end{array}$ & $\begin{array}{l}162 \text { dry eye } \\
\text { disease }\end{array}$ & 16 weeks & $\begin{array}{c}\text { CyA } \\
\text { Placebo }\end{array}$ & $\begin{array}{c}\text { CyA effective on signs and symptoms } \\
\text { of xerophtalmia }\end{array}$ \\
\hline $\begin{array}{l}\text { Sall } \\
{[167]}\end{array}$ & 2000 & $\mathrm{RCT}$ & $\begin{array}{c}\text { Topical } \\
0.05 \%- \\
0.1 \% \\
\end{array}$ & $\begin{array}{l}877 \text { dry eye } \\
\text { disease }\end{array}$ & 6 months & $\begin{array}{c}\text { CyA } \\
\text { Placebo }\end{array}$ & $\begin{array}{c}\text { CyA effective on objective and } \\
\text { subjective measures of xerophtalmia }\end{array}$ \\
\hline $\begin{array}{l}\text { Barber } \\
{[166]}\end{array}$ & 2005 & $\mathrm{CS}$ & $\begin{array}{c}\text { Topical } \\
0.1 \%\end{array}$ & $\begin{array}{l}412 \text { dry eye } \\
\text { disease }\end{array}$ & 3 years & CyA & $\begin{array}{c}\text { CyA safe and effective on } \\
\text { xerophtalmia }\end{array}$ \\
\hline $\begin{array}{l}\text { Roberts } \\
{[168]}\end{array}$ & 2007 & $\mathrm{RCT}$ & $\begin{array}{l}\text { Topical } \\
0.05 \%\end{array}$ & $\begin{array}{l}30 \text { dry eye } \\
\text { disease }\end{array}$ & 6 months & $\begin{array}{c}\text { CyA } \\
\text { Punctual occlusion } \\
\text { CyA + punctual } \\
\text { occlusion }\end{array}$ & $\begin{array}{c}\text { CyA effective on ocular surface } \\
\text { health, additive effects with punctual } \\
\text { occlusion }\end{array}$ \\
\hline $\begin{array}{l}\text { Kim } \\
{[169]}\end{array}$ & 2009 & $\mathrm{RCT}$ & $\begin{array}{l}\text { Topical } \\
0.05 \%\end{array}$ & $\begin{array}{c}150 \text { dry eye } \\
\text { disease }\end{array}$ & 3 months & $\begin{array}{c}\text { CyA } \\
\text { Vitamin A eye drops }\end{array}$ & $\begin{array}{c}\text { CyA as effective as vitamin A eye } \\
\text { drops for dry eye disease }\end{array}$ \\
\hline
\end{tabular}

CyA: cyclosporine A; SSj: Sjogren's syndrome; CS: case-series; CC: case-control; RCT: randomized controlled trial. 
Table 10. Studies on cyclosporine A in macrophage activation syndrome associated with systemic juvenile idiopathic arthritis.

\begin{tabular}{|c|c|c|c|c|c|}
\hline Author [Ref] & Year & $\begin{array}{l}\text { Study } \\
\text { design }\end{array}$ & $\mathrm{N}$ of patients & Treatment group(s) & Outcome \\
\hline $\begin{array}{l}\text { Mouy } \\
{[179]}\end{array}$ & 1996 & CS & 7 & CyA & CyA effective as first line or second line tool in MAS \\
\hline $\begin{array}{l}\text { Ravelli } \\
{[180]}\end{array}$ & 1996 & $\mathrm{CR}$ & 1 & CyA & CyA effective as second line tool in MAS \\
\hline $\begin{array}{l}\text { Stephan } \\
{[181]}\end{array}$ & 2001 & $\mathrm{CS}$ & 18 & $\begin{array}{c}\text { CyA } \\
\text { IvIg } \\
\text { Steroids } \\
\end{array}$ & $\begin{array}{l}\text { CyA effective as first and second line tool in MAS } \\
\text { associated with sJIA }\end{array}$ \\
\hline $\begin{array}{l}\text { Sawhney } \\
\text { [182] }\end{array}$ & 2001 & $\mathrm{CS}$ & 7 & $\begin{array}{c}\text { CyA + steroids } \\
\text { CTX + steroids } \\
\text { Anti-thymocyte } \\
\text { globulin + steroids }\end{array}$ & CyA + steroids effective in MAS \\
\hline $\begin{array}{l}\text { Kounami } \\
{[183]}\end{array}$ & 2005 & $\mathrm{CS}$ & 5 & $\mathrm{CyA}$ & CyA effective as second line tool in MAS \\
\hline $\begin{array}{l}\text { Cortis } \\
{[184]} \\
\end{array}$ & 2006 & $\mathrm{CS}$ & 9 & $\begin{array}{c}\text { CyA + steroids } \\
\text { ETN }\end{array}$ & CyA + steroids effective in MAS \\
\hline $\begin{array}{l}\text { You } \\
{[185]}\end{array}$ & 2006 & $\mathrm{CR}$ & 1 & $\mathrm{CyA}$ & CyA effective as second line tool in MAS \\
\hline $\begin{array}{l}\text { Lin } \\
{[186]}\end{array}$ & 2012 & CS & 4 & $\begin{array}{l}\text { CyA + steroids } \\
\text { IvIg + steroids }\end{array}$ & CyA + steroids effective in MAS \\
\hline
\end{tabular}

CyA: cyclosporine A; IvIg: intra-venous Immunoglobulins; CTX: cyclophosphamide; ETN: etanercept; CS: case-series; CC: case-control; CR: case-report; MAS: macrophage activation syndrome. 
Table 11. The toxicity of cyclosporine A in patients with rheumatoid arthritis [207].

\begin{tabular}{|c|c|}
\hline Toxicity & Frequency $(\%) *$ \\
\hline $\begin{array}{l}\text { Constitutional symptoms } \\
\text { Fatigue } \\
\text { Fever } \\
\text { Influenza-like symptoms } \\
\text { Arthralgias/arthritis }\end{array}$ & $\begin{array}{l}3-12 \% \\
0-4 \% \\
0-6 \% \\
0-5 \%\end{array}$ \\
\hline $\begin{array}{l}\text { Reno-urinary toxicity } \\
\text { Creatine elevation }>30 \% \\
\text { Creatinine elevation }>50 \% \\
\text { Dysuria } \\
\text { Urinary tract infection }\end{array}$ & $\begin{array}{l}13-55 \% \\
3-26 \% \\
0-11 \% \\
0-19 \%\end{array}$ \\
\hline $\begin{array}{l}\text { Cardiovascular toxicity } \\
\text { Hypertension } \\
\text { Arrythmias } \\
\text { Chest pain }\end{array}$ & $\begin{array}{l}2-26 \% \\
1-6 \% \\
1-6 \%\end{array}$ \\
\hline $\begin{array}{l}\text { Gastrointestinal toxicity } \\
\text { Gum hyperplasia } \\
\text { Gingivitis } \\
\text { Stomatitis } \\
\text { Nausea } \\
\text { Vomiting } \\
\text { Dyspepsia } \\
\text { Anorexia } \\
\text { Diarrhea } \\
\text { Abdominal pain }\end{array}$ & $\begin{array}{l}1-4 \% \\
0-4 \% \\
5-16 \% \\
14-24 \% \\
5-14 \% \\
4-12 \% \\
0-3 \% \\
8-18 \% \\
7-15 \%\end{array}$ \\
\hline $\begin{array}{l}\text { Skin and appendage toxicity } \\
\text { Hypertrichosis } \\
\text { Alopecia } \\
\text { Bullous eruption } \\
\text { Rash } \\
\text { Purpura }\end{array}$ & $\begin{array}{l}0-19 \% \\
0-4 \% \\
0-4 \% \\
7-12 \% \\
0-4 \%\end{array}$ \\
\hline $\begin{array}{l}\text { Nervous system toxicity } \\
\text { Dizziness } \\
\text { Headache } \\
\text { Migraine } \\
\text { Paresthesia } \\
\text { Depression } \\
\text { Insomnia }\end{array}$ & $\begin{array}{l}3-8 \% \\
9-25 \% \\
0-3 \% \\
1-11 \% \\
1-6 \% \\
1-4 \%\end{array}$ \\
\hline $\begin{array}{l}\text { Respiratory toxicity } \\
\text { Rhinitis }\end{array}$ & $0-10 \%$ \\
\hline
\end{tabular}




\begin{tabular}{|l|l|}
\hline Sinusitis & $3-8 \%$ \\
Pharingitis & $3-6 \%$ \\
Coughing & $3-7 \%$ \\
Dyspnea & $1-5 \%$ \\
Bronchitis & $0-3 \%$ \\
Pneumonia & $0-4 \%$ \\
\hline
\end{tabular}

*: considering controlled clinical trials. 
Table 12. The interaction of cyclosporine A with other pharmacological compounds.

\begin{tabular}{|l|l|l|}
\hline Increased CyA blood levels & Decreased CyA blood levels & Enhanced nephrotoxicity \\
\hline Allopurinol & & \\
Antimalarials & & \\
Bosentan & Barbiturates & \\
Clarythromycin & Carbamazepin & Aminoglycosides \\
Diltiazem & Rifampin & Amphotericin B \\
Erythromycin & Rifabutin & Ciprofloxacin \\
Fluconazole & Isoniazid & Sulfonamides \\
Imatinib & Nafcillin & ACE inhibitors \\
Ketoconazole & Sulfasalazine & \\
Methylprednisolone (High dose) & & \\
Methotrexate & & \\
Nicardipine & & \\
Verapamil & & \\
\hline
\end{tabular}




\section{References}

1. Ferraccioli GF, Bambara LM, Ferraris M, Perpignano G, Cattaneo R, Porzio F, Accardo S, Mattara L, Zoppini A, Benucci M, Ostuni PA, Pasero G (1997) Effects of cyclosporin on joint damage in rheumatoid arthritis. The Italian Rheumatologists Study Group on Rheumatoid Arthritis. Clin Exp Rheumatol 15:S83-S89.

2. Amor KT, Ryan C, Menter A (2010) The use of cyclosporine in dermatology: Part I. J Am Acad Dermatol 63:925-946.

3. Zhang X, Cui J, Qiu M-C, Li D-Q, Zhang P, Zhang J-S (2008) The effects of cyclosporine A on immunoglobulins deposition in retina of streptozotocin-induced diabetic rats. Zhonghua Nei Ke Za Zhi 47:125-128.

4. Singh JA, Furst DE, Bharat A, Curtis JR, Kavanaugh AF, Kremer JM, Moreland LW, O'Dell J, Winthrop KL, Beukelman T, Bridges SL Jr, Chatham WW, Paulus HE, SuarezAlmazor M, Bombardier C, Dougados M, Khanna D, King CM, Leong AL, Matteson EL, Schousboe JT, Moynihan E, Kolba KS, Jain A, Volkmann ER, Agrawal H, Bae S, Mudano AS, Patkar NM, Saag KG (2012) 2012 Update of the 2008 American College of Rheumatology recommendations for the use of disease-modifying antirheumatic drugs and biologic agents in the treatment of rheumatoid arthritis. Arthritis Care Res 64:625-639.

5. Dougados M, Amor B (1987) Cyclosporin A in rheumatoid arthritis: preliminary clinical results of an open trial. Arthritis Rheum 30:83-87.

6. Dougados M, Duchesne L, Awada H, Amor B (1989) Assessment of efficacy and acceptability of low dose cyclosporin in patients with rheumatoid arthritis. Ann Rheum Dis 48:550-556.

7. Weinblatt ME, Coblyn JS, Fraser PA, Anderson RJ, Spragg J, Trentham DE, Austen KF (1987) Cyclosporin A treatment of refractory rheumatoid arthritis. Arthritis Rheum 30:1117.

8. Madhok R, Torley HI, Capell HA (1991) A study of the longterm efficacy and toxicity of cyclosporine A in rheumatoid arthritis. J Rheumatol18:1485-1489.

9. Tugwell P, Bombardier C, Gent M, Bennett KJ, Bensen WG, Carette S, Chalmers A, Esdaile JM, Klinkhoff AV, Kraag GR, et al (1990) Low-dose cyclosporin versus placebo in patients with rheumatoid arthritis. Lancet 335:1051-1055.

10. van Rijthoven AW, Dijkmans BA, Thè HS, Meijers KA, Montnor-Beckers ZL, Moolenburgh JD, Boers M, Cats A (1991) Comparison of cyclosporine and Dpenicillamine for rheumatoid arthritis: a randomized, double blind, multicenter study. J Rheumatol 18:815-820.

11. Drosos AA, Voulgari PV, Katsaraki A, Zikou AK (2000) Influence of cyclosporin A on radiological progression in early rheumatoid arthritis patients: a 42-month prospective study. Rheumatol Int 19:113-118.

12. Pasero G, Priolo F, Marubini E, Fantini F, Ferraccioli G, Magaro M, Marcolongo R, Oriente P, Pipitone V, Portioli I, Tirri G, Trotta F, Della Casa-Alberighi O (1996) Slow progression of joint damage in early rheumatoid arthritis treated with cyclosporin $\mathrm{A}$. Arthritis Rheum 39:1006-1015. 
13. Førre O (1994) Radiologic evidence of disease modification in rheumatoid arthritis patients treated with cyclosporine. Results of a 48-week multicenter study comparing low-dose cyclosporine with placebo. Norwegian Arthritis Study Group. Arthritis Rheum 37:15061512.

14. Zeidler HK, Kvien TK, Hannonen P, Wollheim FA, Førre O, Geidel H, Hafström I, Kaltwasser JP, Leirisalo-Repo M, Manger B, Laasonen L, Markert ER, Prestele H, Kurki P (1998) Progression of joint damage in early active severe rheumatoid arthritis during 18 months of treatment: comparison of low-dose cyclosporin and parenteral gold. $\mathrm{Br} \mathrm{J}$ Rheumatol 37:874-882.

15. Kvien TK, Zeidler HK, Hannonen P, Wollheim FA, Førre O, Hafström I, Kaltwasser JP, Leirisalo-Repo M, Manger B, Laasonen L, Prestele H, Kurki P (2002) Long term efficacy and safety of cyclosporin versus parenteral gold in early rheumatoid arthritis: a three year study of radiographic progression, renal function, and arterial hypertension. Ann Rheum Dis 61:511-516.

16. van Rijthoven AW, Dijkmans BA, Goei Thé HS, Boers M, Cats A (1991) Longterm cyclosporine therapy in rheumatoid arthritis. J Rheumatol 18:19-23.

17. Krüger K, Schattenkirchner M (1994) Comparison of cyclosporin A and azathioprine in the treatment of rheumatoid arthritis--results of a double-blind multicentre study. Clin Rheumatol 13:248-255.

18. Ahern MJ, Harrison W, Hollingsworth P, Bradley J, Laing B, Bayliss C (1991) A randomised double-blind trial of cyclosporin and azathioprine in refractory rheumatoid arthritis. Aust N Z J Med 21:844-849.

19. Førre O, Bjerkhoel F, Salvesen CF, Berg KJ, Rugstad HE, Saelid G, Mellbye OJ, Kåss E (1987) An open, controlled, randomized comparison of cyclosporine and azathioprine in the treatment of rheumatoid arthritis: a preliminary report. Arthritis Rheum 30:88-92.

20. Bakker MF, Jacobs JWG, Welsing PMJ, van der Werf JH, Linn-Rasker SP, van der Veen MJ, Lafeber FP, Bijlsma JW; Utrecht Arthritis Cohort Study Group (2010) Are switches from oral to subcutaneous methotrexate or addition of ciclosporin to methotrexate useful steps in a tight control treatment strategy for rheumatoid arthritis? A post hoc analysis of the CAMERA study. Ann Rheum Dis 69:1849-1852.

21. Drosos AA, Voulgari PV, Papadopoulos IA, Politi EN, Georgiou PE, Zikou AK (1998) Cyclosporine $\mathrm{A}$ in the treatment of early rheumatoid arthritis. A prospective, randomized 24-month study. Clin Exp Rheumatol 16:695-701.

22. Marchesoni A, Battafarano N, Arreghini M, Pellerito R, Cagnoli M, Prudente P, Cerase A, Priolo F, Tosi S (2002) Step-down approach using either cyclosporin A or methotrexate as maintenance therapy in early rheumatoid arthritis. Arthritis Rheum 47:59-66.

23. Sarzi-Puttini P, D'Ingianna E, Fumagalli M, Scarpellini M, Fiorini T, Chérié-Lignière EL, Panni B, Fiorentini F, Corbelli V, Beyene NB, Mastaglio C, Severi C, Locati M, Cazzola M, Menozzi G, Monti G, Saccardo F, Alfieri G, Atzeni F (2005) An open, randomized comparison study of cyclosporine A, cyclosporine A + methotrexate and cyclosporine A + hydroxychloroquine in the treatment of early severe rheumatoid arthritis. Rheumatol Int 25:15-22. 
24. Miranda JM, Alvarez-Nemegyei J, Saavedra MA, Terán L, Galván-Villegas F, GarcíaFigueroa J, Jara LJ, Barile L; GRECIA Group (2004) A randomized, double-blind, multicenter, controlled clinical trial of cyclosporine plus chloroquine vs. cyclosporine plus placebo in early-onset rheumatoid arthritis. Arch Med Res 35:36-42.

25. Bendix G, Bjelle A (1996) Adding low-dose cyclosporin A to parenteral gold therapy in rheumatoid arthritis: a double-blind placebo-controlled study. Br J Rheumatol 35:11421149.

26. van den Borne BE, Landewé RB, Goei Thé HS, ietveld JH, Zwinderman AH, Bruyn GA, Breedveld FC, Dijkmans BA (1998) Combination therapy in recent onset rheumatoid arthritis: a randomized double blind trial of the addition of low dose cyclosporine to patients treated with low dose chloroquine. J Rheumatol 25:1493-1498.

27. Gerards AH, Landewé RBM, Prins APA, Bruyn GA, Goei Thé HS, Laan RF, Dijkmans BA (2003) Cyclosporin A monotherapy versus cyclosporin A and methotrexate combination therapy in patients with early rheumatoid arthritis: a double blind randomised placebo controlled trial. Ann Rheum Dis 62:291-296.

28. Bensen W, Tugwell P, Roberts RM, Ludwin D, Ross H, Grace E, Gent M (1994) Combination therapy of cyclosporine with methotrexate and gold in rheumatoid arthritis (2 pilot studies). J Rheumatol 21:2034-2038.

29. Salaffi F, Carotti M, Cervini C (1996) Combination therapy of cyclosporine A with methotrexate or hydroxychloroquine in refractory rheumatoid arthritis. Scand J Rheumatol 25:16-23.

30. Tugwell P, Pincus T, Yocum D, Stein M, Gluck O, Kraag G, McKendry R, Tesser J, Baker P, Wells G (1996) Combination therapy with cyclosporine and methotrexate in severe rheumatoid arthritis. The Methotrexate-Cyclosporine Combination Study Group. N Engl J Med 333:137-141.

31. Karanikolas G, Charalambopoulos D, Andrianakos A, Antoniades C, Katsilambros N (2006) Combination of cyclosporine and leflunomide versus single therapy in severe rheumatoid arthritis. J Rheumatol 33:486-489.

32. Hetland ML, Stengaard-Pedersen K, Junker P, Lottenburger T, Ellingsen T, Andersen LS, Hansen I, Skjødt H, Pedersen JK, Lauridsen UB, Svendsen A, Tarp U, Pødenphant J, Hansen G, Lindegaard H, de Carvalho A, Østergaard M, Hørslev-Petersen K; CIMESTRA Study Group (2006) Combination treatment with methotrexate, cyclosporine, and intraarticular betamethasone compared with methotrexate and intraarticular betamethasone in early active rheumatoid arthritis: an investigator-initiated, multicenter, randomized, double-blind, parallel-group, placebo-controlled study. Arthritis Rheum 54:1401-1409.

33. Marchesoni A, Battafarano N, Arreghini M, Panni B, Gallazzi M, Tosi S (2003) Radiographic progression in early rheumatoid arthritis: a 12-month randomized controlled study comparing the combination of cyclosporin and methotrexate with methotrexate alone. Rheumatology (Oxford) 42:1545-1549.

34. Choy EHS, Smith CM, Farewell V, Walker D, Hassell A, Chau L, Scott DL; CARDERA (Combination Anti-Rheumatic Drugs in Early Rheumatoid Arhritis) Trial Group (2007) Factorial randomised controlled trial of glucocorticoids and combination disease modifying drugs in early rheumatoid arthritis. Ann Rheum Dis 67:656-663. 
35. Hetland ML, Stengaard-Pedersen K, Junker P, Lottenburger T, Ellingsen T, Andersen LS, Hansen I, Skjødt H, Pedersen JK, Lauridsen UB, Svendsen A, Tarp U, Pødenphant J, Hansen G, Lindegaard H, de Carvalho A, Østergaard M, Hørslev-Petersen K; CIMESTRA Study Group (2010) Radiographic progression and remission rates in early rheumatoid arthritis - MRI bone oedema and anti-CCP predicted radiographic progression in the 5-year extension of the double-blind randomised CIMESTRA trial. Ann Rheum Dis 69:17891795.

36. Stein CM, Pincus T, Yocum D, Tugwell P, Wells G, Gluck O, Kraag G, Torley H, Tesser J, McKendry R, Brooks RH (1997) Combination treatment of severe rheumatoid arthritis with cyclosporine and methotrexate for forty-eight weeks: an open-label extension study. The Methotrexate-Cyclosporine Combination Study Group. Arthritis Rheum 40:1843-1851.

37. Ferraccioli GF, Gremese E, Tomietto P, Favret G, Damato R, Di Poi E (2002) Analysis of improvements, full responses, remission and toxicity in rheumatoid patients treated with step-up combination therapy (methotrexate, cyclosporin A, sulphasalazine) or monotherapy for three years. Rheumatology (Oxford). 41:892-898.

38. Sidiropoulos PI, Siakka P, Raptopoulou A, Mamoulaki M, Choulaki C, Koutala H, Kouroumali H, Kritikos H, Boumpas DT (2006) An open label, single dose study to evaluate the safety, efficacy, and effects on CD25 expression of ciclosporin in patients with active rheumatoid arthritis despite treatment with methotrexate and infliximab. Ann Rheum Dis 65:538-541.

39. Temekonidis TI, Georgiadis AN, Alamanos Y, Bougias DV, Voulgari PV, Drosos AA (2002) Infliximab treatment in combination with cyclosporin A in patients with severe refractory rheumatoid arthritis. Ann Rheum Dis 61:822-825.

40. Migliore A, Bizzi E, Massafra U, Vacca F, Martin Martin LS, Ferlito C, Podesta E, Granata M, Lagana B (2011) A new chance to maintain remission induced by anti-TNF agents in rheumatoid arthritis patients: CYnAR study II of a 12-month follow-up. Int J Immunopathol Pharmacol 24:167-74.

41. Picchianti Diamanti A, Argento G, Podestà E, Germano V, Fazi S, Migliore A, David V, D'Amelio R, Laganà B (2012) Can the association of Ciclosporine A and Methotrexate maintain remission/low disease activity induced by etanercept in early Rheumatoid Arthritis patients? Evaluation by Magnetic Resonance Imaging. Int J Immunopathol Pharmacol 25:301-305.

42. Hochberg MC, Tracy JK, Flores RH (2001) "Stepping-up” from methotrexate: a systematic review of randomised placebo controlled trials in patients with rheumatoid arthritis with an incomplete response to methotrexate. Ann Rheum Dis 60:iii51-iii54.

43. Helliwell PS, Taylor WJ, CASPAR Study Group (2008) Treatment of psoriatic arthritis and rheumatoid arthritis with disease modifying drugs -- comparison of drugs and adverse reactions. J Rheumatol 35:472-476.

44. Hernández GL, Volpert OV, Iñiguez MA, Lorenzo E, Martínez-Martínez S, Grau R, Fresno M, Redondo JM (2001) Selective inhibition of vascular endothelial growth factor-mediated angiogenesis by cyclosporin $\mathrm{A}$ : roles of the nuclear factor of activated $\mathrm{T}$ cells and cyclooxygenase 2. J Exp Med 193:607-620.

45. Cho M-L, Cho C-S, Min S-Y, Kim SH, Lee SS, Kim WU, Min DJ, Min JK, Youn J, 
Hwang SY, Park SH, Kim HY (2002) Cyclosporine inhibition of vascular endothelial growth factor production in rheumatoid synovial fibroblasts. Arthritis Rheum 46:12021209.

46. Ritchlin CT, Kavanaugh A, Gladman DD, Mease PJ, Helliwell P, Boehncke WH, de Vlam K, Fiorentino D, Fitzgerald O, Gottlieb AB, McHugh NJ, Nash P, Qureshi AA, Soriano ER, Taylor WJ; Group for Research and Assessment of Psoriasis and Psoriatic Arthritis (GRAPPA) (2009) Treatment recommendations for psoriatic arthritis. Ann Rheum Dis 68:1387-1394.

47. Gupta AK, Matteson EL, Ellis CN, Ho VC, Tellner DC, Voorhees JJ, McCune WJ (1989) Cyclosporine in the treatment of psoriatic arthritis. Arch Dermatol 125:507-510.

48. Steinsson K, Jónsdóttir I, Valdimarsson H (1990) Cyclosporin A in psoriatic arthritis: an open study. Ann Rheum Dis 49:603-606.

49. Salvarani C, Macchioni P, Boiardi L, Rossi F, Casadei Maldini M, Mancini R, Beltrandi E, Lodi L, Bisighini G, Portioli I (1992) Low dose cyclosporine A in psoriatic arthritis: relation between soluble interleukin 2 receptors and response to therapy. J Rheumatol 19:74-79.

50. Mahrle G, Schulze HJ, Bräutigam M, Mischer P, Schopf R, Jung EG, Weidinger G, Färber L (1996) Anti-inflammatory efficacy of low-dose cyclosporin A in psoriatic arthritis. A prospective multicentre study. Br J Dermatol 135:752-757.

51. Spadaro A, Riccieri V, Sili-Scavalli A, Sensi F, Taccari E, Zoppini A (1995) Comparison of cyclosporin A and methotrexate in the treatment of psoriatic arthritis: a one-year prospective study. Clin Exp Rheumatol 13:589-593.

52. Salvarani C, Macchioni P, Olivieri I, Marchesoni A, Cutolo M, Ferraccioli G, Cantini F, Salaffi F, Padula A, Lovino C, Dovigo L, Bordin G, Davoli C, Pasero G, Alberighi OD (2001) A comparison of cyclosporine, sulfasalazine, and symptomatic therapy in the treatment of psoriatic arthritis. J Rheumatol 28:2274-2282.

53. Fraser AD, van Kuijk AWR, Westhovens R, Karim Z, Wakefield R, Gerards AH, Landewé R, Steinfeld SD, Emery P, Dijkmans BA, Veale DJ (2005) A randomised, double blind, placebo controlled, multicentre trial of combination therapy with methotrexate plus ciclosporin in patients with active psoriatic arthritis. Ann Rheum Dis 64:859-864.

54. D'Angelo S, Cutro MS, Lubrano E, Leccese P, Mennillo GA, Ferrara N, Olivieri I (2010) Combination therapy with ciclosporin and etanercept in patients with psoriatic arthritis. Ann Rheum Dis 69:934-935.

55. Atzeni F, Boccassini L, Antivalle M, Salaffi F, Sarzi-Puttini P (2011) Etanercept plus ciclosporin versus etanercept plus methotrexate for maintaining clinical control over psoriatic arthritis: a randomised pilot study. Ann Rheum Dis 70:712-714.

56. Sarzi-Puttini P, Cazzola M, Panni B, Turiel M, Fiorini T, Belai-Beyene N, Chèrié-Lignièré EL (2002) Long-term safety and efficacy of low-dose cyclosporin A in severe psoriatic arthritis. Rheumatol Int 21:234-238.

57. Macchioni P, Boiardi L, Cremonesi T, Battistel B, Casadei-Maldini M, Beltrandi E, Mancini R, Salvarani C (1998) The relationship between serum-soluble interleukin-2 
receptor and radiological evolution in psoriatic arthritis patients treated with cyclosporin-A. Rheumatol Int 18:27-33.

58. Israel-Biet D, Noël L-H, Bach MA, Dardenne M, Bach JF (1983) Marked reduction of DNA antibody production and glomerulopathy in thymulin (FTS-Zn) or cyclosporin A treated (NZB X NZW) F1 mice. Clin Exp Immunol 54:359-365.

59. Jones MG, Harris G (1985) Prolongation of life in female NZB/NZW (F1) hybrid mice by cyclosporin A. Clin Exp Immunol 59:1-9.

60. Gunn HC (1986) Successful treatment of autoimmunity in (NZB X NZW)F1 mice with cyclosporin and (Nva2)-cyclosporin: I. Reduction of autoantibodies. Clin Exp Immunol 64:225-233.

61. Isenberg DA, Snaith ML, Morrow WJ, Al-Khader AA, Cohen SL, Fisher C, Mowbray J (1981) Cyclosporin A for the treatment of systemic lupus erythematosus. Int $\mathrm{J}$ Immunopharmacol 3:163-169.

62. Feutren G, Querin S, Noël LH, Chatenoud L, Beaurain G, Tron F, Lesavre P, Bach JF (1987) Effects of cyclosporine in severe systemic lupus erythematosus. J Pediatr 111:10631068.

63. Miescher PA, Favre H, Chatelanat F, Mihatsch MJ (1987) Combined steroid-cyclosporin treatment of chronic autoimmune diseases. Clinical results and assessment of nephrotoxicity by renal biopsy. Klin Wochenschr 65:727-736.

64. Tokuda M, Kurata N, Mizoguchi A, Inoh M, Seto K, Kinashi M, Takahara J (1994) Effect of low-dose cyclosporin A on systemic lupus erythematosus disease activity. Arthritis Rheum 37:551-558.

65. Caccavo D, Laganà B, Mitterhofer AP, Ferri GM, Afeltra A, Amoroso A, Bonomo L (1997) Long-term treatment of systemic lupus erythematosus with cyclosporin A. Arthritis Rheum 40:27-35.

66. Dostál C, Tesar V, Rychlik I, Zabka J, Vencovský J, Bartûnková J, Stejskalová A, Tegzova D (1998) Effect of 1 year cyclosporine A treatment on the activity and renal involvement of systemic lupus erythematosus: a pilot study. Lupus 7:29-36.

67. Miescher PA, Favre H, Lemoine R, Huang YP (1994) Drug combination therapy of systemic lupus erythematosus. Springer Semin Immunopathol 16:295-311.

68. Dammacco F, Casa Alberighi Della O, Ferraccioli G, Racanelli V, Casatta L, Bartoli E (2000) Cyclosporine-A plus steroids versus steroids alone in the 12-month treatment of systemic lupus erythematosus. Int J Clin Lab Res 30:67-73.

69. Morton SJ, Powell RJ (2000) An audit of cyclosporin for systemic lupus erythematosus and related overlap syndromes: limitations of its use. Ann Rheum Dis 59:487-489.

70. Xiong W, Lahita RG (2013) Pragmatic approaches to therapy for systemic lupus erythematosus. Nat Rev Rheumatol 10:97-107.

71. Bensman A, Niaudet P (2010) Non-immunologic mechanisms of calcineurin inhibitors explain its antiproteinuric effects in genetic glomerulopathies. Pediatr Nephrol 25:1197- 
72. Faul C, Donnelly M, Merscher-Gomez S, Chang YH, Franz S, Delfgaauw J, Chang JM, Choi HY, Campbell KN, Kim K, Reiser J, Mundel P (2008) The actin cytoskeleton of kidney podocytes is a direct target of the antiproteinuric effect of cyclosporine A. Nat Med $14: 931-938$.

73. Favre H, Miescher PA, Huang YP, Chatelanat F, Mihatsch MJ (1989) Ciclosporin in the treatment of lupus nephritis. Am J Nephrol 9:57-60.

74. Hussein MM, Mooij JM, Roujouleh H (1993) Cyclosporine in the treatment of lupus nephritis including two patients treated during pregnancy. Clin Nephrol 40:160-163.

75. Manger K, Kalden JR, Manger B (1996) Cyclosporin A in the treatment of systemic lupus erythematosus: results of an open clinical study. Br J Rheumatol 35:669-675.

76. Tam LS, Li EK, Leung CB, Wong KC, Lai FM, Wang A, Szeto CC, Lui SF (1998) Longterm treatment of lupus nephritis with cyclosporin A. QJM 91:573-580.

77. Rihova Z, Vankova Z, Maixnerova D, Dostal C, Jancova E, Honsova E, Merta M, Rysava R, Tesar V (2007) Treatment of lupus nephritis with cyclosporine - an outcome analysis. Kidney Blood Press Res 30:124-8.

78. Ogawa H, Kameda H, Nagasawa H, Sekiguchi N, Takei H, Tsuzaka K, Amano K, Takeuchi T (2007) Prospective study of low-dose cyclosporine A in patients with refractory lupus nephritis. Mod Rheumatol 17:92-97.

79. Ogawa H, Kameda H, Amano K, Takeuchi T (2010) Efficacy and safety of cyclosporine A in patients with refractory systemic lupus erythematosus in a daily clinical practice. Lupus 19:162-169.

80. Kamijo Y, Hashimoto K, Takahashi K, Ehara T, Shigematsu H, Higuchi M (2011) Treatment with cyclosporine A improves SLE disease activity of Japanese patients with diffuse proliferative lupus nephritis. Clin Nephrol 76:136-143.

81. Balletta M, Sabella D, Magri P, Sepe V, Stanziale P, Di Luccio R, Colucci G, Fuiano G (1992) Ciclosporin plus steroids versus steroids alone in the treatment of lupus nephritis. Contrib Nephrol 99:129-130.

82. Moroni G, Doria A, Mosca M, Alberighi OD, Ferraccioli G, Todesco S, Manno C, Altieri P, Ferrara R, Greco S, Ponticelli C (2006) A randomized pilot trial comparing cyclosporine and azathioprine for maintenance therapy in diffuse lupus nephritis over four years. Clin $\mathrm{J}$ Am Soc Nephrol 1:925-932.

83. Zavada J, Pesickova S, Rysava R, Olejarova M, Horák P, Hrncír Z, Rychlík I, Havrda M, Vítova J, Lukác J, Rovensky J, Tegzova D, Böhmova J, Zadrazil J, Hána J, Dostál C, Tesar V (2010) Cyclosporine A or intravenous cyclophosphamide for lupus nephritis: the Cyclofa-Lune study. Lupus 19:1281-9.

84. Zavada J, Pesickova SS, Rysava R, Horák P, Hrncír Z, Lukác J, Rovensky J, Vítová J, Havrda M, Rychlík I, Böhmova J, Vlasáková V, Slatinská J, Zadrazil J, Olejárová M, Tegzova D, Tesar V (2013) Extended follow-up of the CYCLOFA-LUNE trial comparing two sequential induction and maintenance treatment regimens for proliferative lupus 
nephritis based either on cyclophosphamide or on cyclosporine A. Lupus 23:69-74.

85. Moroni G, Doria A, Ponticelli C (2009) Cyclosporine (CsA) in lupus nephritis: assessing the evidence. Nephrol Dial Transplant 24:15-20.

86. Radhakrishnan J, Kunis CL, D'Agati V, Appel GB (1994) Cyclosporine treatment of lupus membranous nephropathy. Clin Nephrol 42:147-154.

87. Hallegua D, Wallace DJ, Metzger AL, Rinaldi RZ, Klinenberg JR (2000) Cyclosporine for lupus membranous nephritis: experience with ten patients and review of the literature. Lupus 9:241-251.

88. Tam LS, Li EK, Szeto CC, Wong SM, Leung CB, Lai FM, Wong KC, Lui SF (2001) Treatment of membranous lupus nephritis with prednisone, azathioprine and cyclosporin A. Lupus 10:827-829.

89. Hu W, Liu Z, Shen S, Li S, Yao X, Chen H, Li L (2003) Cyclosporine A in treatment of membranous lupus nephropathy. Chin Med J 116:1827-1830.

90. Austin HA, Illei GG, Braun MJ, Balow JE (2009) Randomized, controlled trial of prednisone, cyclophosphamide, and cyclosporine in lupus membranous nephropathy. J Am Soc Nephrol 20:901-911.

91. Yang M, Li M, He W, Wang B, Gu Y (2014) Calcineurin inhibitors may be a reasonable alternative to cyclophosphamide in the induction treatment of active lupus nephritis: A systematic review and meta-analysis. Exp Ther Med 2014:1-8.

92. Shaharir SS, Mustafar R, Mohd R, Mohd Said MS, Gafor HA (2015) Persistent hypertension in lupus nephritis and the associated risk factors. Clin Rheumatol 34:93-97.

93. Quartuccio L, Sacco S, Franzolini N, Perin A, Ferraccioli G, De Vita S (2006) Efficacy of cyclosporin-A in the long-term management of thrombocytopenia associated with systemic lupus erythematosus. Lupus 15:76-79.

94. Sugiyama M, Ogasawara H, Kaneko H, Hishikawa T, Sekigawa I, Iida N, Hashimoto H, Hirose S (1998) Effect of extremely low dose cyclosporine treatment on the thrombocytopenia in systemic lupus erythematosus. Lupus 7:53-6.

95. Wang SW, Cheng TT (2005) Systemic lupus erythematosus with refractory hemolytic anemia effectively treated with cyclosporin A: a case report. Lupus 14:483-485.

96. Singh NP, Prakash A, Garg D, Makhija A, Pathania A, Prakash N, Kubba S, Agarwal SK (2004) Aplastic anemia complicating systemic lupus erythematosus: successful management with cyclosporine. Rheumatol Int 24:40-42.

97. Atzeni F, Sarzi-Puttini P, Capsoni F, Vulpio L, Carrabba M (2003) Successful treatment of pure red cell aplasia in systemic lupus erythematosus with cyclosporin A. Clin Exp Rheumatol 21:759-62.

98. Duarte-Salazar C, Cazarín-Barrientos J, Goycochea-Robles MV, Collazo-Jaloma J, BurgosVargas R (2000) Successful treatment of pure red cell aplasia associated with systemic lupus erythematosus with cyclosporin A. Rheumatology (Oxford) 39:1155-7. 
99. Arcasoy MO, Chao NJ (2005) T-cell-mediated pure red-cell aplasia in systemic lupus erythematosus: response to cyclosporin A and mycophenolate mofetil. Am J Hematol 78:161-3.

100. Takahashi H, Tsuboi H, Kurata I, Takahashi H, Inoue S, Ebe H, Yokosawa M, Hagiwara S, Hirota T, Asashima H, Kaneko S, Kawaguchi H, Kurashima Y, Miki H, Umeda N, Kondo Y, Ogishima H, Suzuki T, Matsumoto I, Sumida T (2015) Predictors of the response to treatment in acute lupus hemophagocytic syndrome. Lupus 24:659-668.

101. Grabbe S, Kolde G (1995) Coexisting lichen planus and subacute cutaneous lupus erythematosus. Clin Exp Dermatol 20:249-254.

102. Saeki Y, Ohshima S, Kurimoto I, Miura H, Suemura M (2000) Maintaining remission of lupus erythematosus profundus (LEP) with cyclosporin A. Lupus 9:390-392.

103. Heule F, van Joost T, Beukers R (1986) Cyclosporine in the Treatment of Lupus Erythematosus. Arch Dermatol 122:973-974.

104. Yell JA, Burge SM (1994) Cyclosporin and discoid lupus erythematosus. Br J Dermatol 131:132-133.

105. Alevizopoulos A, Dusserre Y, Rüegg U, Mermod N (1997) Regulation of the transforming growth factor beta-responsive transcription factor CTF-1 by calcineurin and calcium/calmodulin-dependent protein kinase IV. J Biol Chem 272:23597-23605.

106. Karashima T, Hachisuka H, Sasai Y (1996) FK506 and cyclosporin A inhibit growth factor-stimulated human keratinocyte proliferation by blocking cells in the G0/G1 phases of the cell cycle. J Dermatol Sci 12:246-254.

107. Frances C, Branchet MC, Blétry O, Lefevre C, Boisnic S, Kern P, Godeau P (1988) Skin collagen from scleroderma patients before and after cyclosporin A treatment. Clin Exp Dermatol 13:1-3.

108. Clements PJ, Lachenbruch PA, Sterz M, Danovitch G, Hawkins R, Ippoliti A, Paulus HE (1993) Cyclosporine in systemic sclerosis. Results of a forty-eight-week open safety study in ten patients. Arthritis Rheum 36:75-83.

109. Zachariae H, Halkier-Sørensen L, Heickendorff L, Zachariae E, Hansen HE (1990) Cyclosporin A treatment of systemic sclerosis. Br J Dermatol 122:677-681.

110. Roch B, Wollina U, Schroeder H-E (2004) Long-term cyclosporin A in systemic sclerosis. J Eur Acad Dermatol Venereol 18:235-236.

111. Filaci G, Cutolo M, Scudeletti M, Castagneto C, Derchi L, Gianrossi R, Ropolo F, Zentilin P, Sulli A, Murdaca G, Ghio M, Indiveri F, Puppo F (1999) Cyclosporin A and iloprost treatment of systemic sclerosis: clinical results and interleukin-6 serum changes after 12 months of therapy. Rheumatology (Oxford) 38:992-996.

112. Zentilin P, Savarino V, Puppo F, Scudeletti M, Indiveri F (1994) Improvement in esophageal motor abnormalities in systemic sclerosis patients treated with cyclosporine: comment on the article by Clements et al. Arthritis Rheum 37:301-302.

113. Ando K, Nakashita T, Kaneko N, Takahashi K, Motojima S (2013) Long-term efficacy and 
safety of maintenance therapy with azathioprine or cyclosporine for interstitial lung disease with diffuse cutaneous scleroderma. Clin Exp Rheumatol 31:191-192.

114. Walker KM, Pope J, Scleroderma Clinical Trials Consortium, Canadian Scleroderma Research Group (2011) Expert agreement on EULAR/EUSTAR recommendations for the management of systemic sclerosis. J Rheumatol 38:1326-1328.

115. Denton CP, Sweny P, Abdulla A, Black CM (1994) Acute renal failure occurring in scleroderma treated with cyclosporin A: a report of three cases. Br J Rheumatol 33:90-92.

116. Grau JM, Herrero C, Casademont J, Fernández-Solà J, Urbano-Márquez A (1994) Cyclosporine A as first choice therapy for dermatomyositis. J Rheumatol 21:381-382.

117. Vencovský J, Jarosová K, Machácek S, Studýnková J, Kafková J, Bartůnková J, Nemcová D, Charvát F (2000) Cyclosporine A versus methotrexate in the treatment of polymyositis and dermatomyositis. Scand J Rheumatol 29:95-102.

118. Mii S, Niiyama S, Kusunoki M, Arai S, Katsuoka K (2006) Cyclosporine A as treatment of esophageal involvement in dermatomyositis. Rheumatol Int 27:183-185.

119. Kotani T, Makino S, Kawasaki Y, Hirano S, Tabushi Y, Kagitani M, Takeuchi T, Hanafusa $\mathrm{T}$ (2005) A case of interstitial pneumonia associated with dermatomyositis effectively treated with cyclosporin-A and cyclophosphamide pulse. Nihon Rinsho Meneki Gakkai Kaishi 28:148-153.

120. Kotani T, Makino S, Takeuchi T, Kagitani M, Shoda T, Hata A, Tabushi Y, Hanafusa T (2008) Early intervention with corticosteroids and cyclosporin A and 2-hour postdose blood concentration monitoring improves the prognosis of acute/subacute interstitial pneumonia in dermatomyositis. J Rheumatol 35:254-259.

121. Terao M, Ozawa K, Inui S, Murota H, Yokomi A, Itami S (2007) A case of dermatomyositis complicated with pneumomediastinum. Mod Rheumatol 17:156-159.

122. Takada K, Nagasaka K, Miyasaka N (2005) Polymyositis/dermatomyositis and interstitial lung disease: a new therapeutic approach with T-cell-specific immunosuppressants. Autoimmunity 38:383-392.

123. Labirua-Iturburu A, Selva-O'Callaghan A, Martínez-Gómez X, Trallero-Araguás E, Labrador-Horrillo M, Vilardell-Tarrés M (2013) Calcineurin inhibitors in a cohort of patients with antisynthetase-associated interstitial lung disease. Clin Exp Rheumatol 31:436-439.

124. Sakamoto S, Homma S, Kawabat M, Kono T, Motoi N, Yoshimur K (2005) A case of corticosteroid-resistant nonspecific interstitial pneumonia associated with dermatomyositis successfully treated with cyclosporin A. Nihon Kokyuki Gakkai Zasshi 43:171-178.

125. Cavagna L, Caporali R, Abdì-Alì L, Dore R, Meloni F, Montecucco C (2013) Cyclosporine in anti-Jo1-positive patients with corticosteroid-refractory interstitial lung disease. J Rheumatol 40:484-492.

126. Suzuki A, Shoji N, Kikuchi E, Uekubo K, Aoki N, Sonoda Y, Torigai H, Yamashita H, Fujita K, Okai T (2013) Successful combination therapy with corticosteroids, biweekly intravenous pulse cyclophosphamide and cyclosporin A for acute interstitial pneumonia in 
patients with dermatomyositis: report of three cases. Nihon Rinsho Meneki Gakkai Kaishi 36:122-128.

127. Ingegnoli F, Lubatti C, Ingegnoli A, Boracchi P, Zeni S, Meroni PL (2012) Interstitial lung disease outcomes by high-resolution computed tomography (HRCT) in Anti-Jo1 antibodypositive polymyositis patients: A single centre study and review of the literature. Autoimmun Rev 11:335-340.

128. Gremmel F, Druml W, Schmidt P, Graninger W (1988) Cyclosporin in Wegener granulomatosis. Ann Intern Med 108:491.

129. Borleffs JC, van der Zwan JC (1990) Cyclosporin in Wegener's granulomatosis with renal failure. Ann Rheum Dis 49:568.

130. Harley N, Ihle B (1990) Wegener's granulomatosis--use of cyclosporin-A: a case report. Aust N Z J Med 20:71-73.

131. Inoue K, Kondo M, Inoue M, Ishino H, Kamitsuji Y, Sano H (2000) Successful treatment with combination therapy of cyclophosphamide and cyclosporin for late recurrence of Wegener granulomatosis. Arch Intern Med 160:393-394.

132. McDermott EM, Powell RJ (1998) Cyclosporin in the treatment of Churg-Strauss syndrome. Ann Rheum Dis 57:258-259.

133. Schaufelberger C, Andersson R, Nordborg E (1998) No additive effect of cyclosporin A compared with glucocorticoid treatment alone in giant cell arteritis: results of an open, controlled, randomized study. Br J Rheumatol 37:464-5.

134. Wendling D, Hory B, Blanc D (1985) Cyclosporine: a new adjuvant therapy for giant cell arteritis? Arthritis Rheum 28:1078-1079.

135. Schaufelberger C, Möllby H, Uddhammar A, Bratt J, Nordborg E (2006) No additional steroid-sparing effect of cyclosporine A in giant cell arteritis. Scand J Rheumatol 35:327329.

136. Horigome H, Kamoda T, Matsui A (1999) Treatment of glucocorticoid-dependent Takayasu's arteritis with cyclosporin. Med J Aust 170:566.

137. Fearfield LA, Ross JR, Farrell AM, Costello C, Bunker CB, Staughton RC (1999) Pyoderma gangrenosum associated with Takayasu's arteritis responding to cyclosporin. $\mathrm{Br}$ J Dermatol 141:339-343.

138. Fullerton SH, Abel EA, Getz K, el-Ramahi K (1991) Cyclosporine treatment of severe recalcitrant pyoderma gangrenosum in a patient with Takayasu's arteritis. Arch Dermatol 127:1731-1732.

139. de Merieux P, Spitler LE, Paulus HE (1981) Treatment of Behcet's syndrome with levamisole. Arthritis Rheum 24:64-70.

140. Hatemi G, Silman A, Bang D, Bodaghi B, Chamberlain AM, Gul A, Houman MH, Kötter I, Olivieri I, Salvarani C, Sfikakis PP, Siva A, Stanford MR, Stübiger N, Yurdakul S, Yazici H (2008) EULAR recommendations for the management of Behçet disease. Ann Rheum Dis 67:1656-1662. 
141. Ozyazgan Y, Yurdakul S, Yazici H, üzün B, Işçimen A, Tüzün Y, Aktunç T, Pazarli H, Hamuryudan V, Müftüoğlu A (1992) Low dose cyclosporin A versus pulsed cyclophosphamide in Behçet's syndrome: a single masked trial. Br J Ophthalmol 76:241243.

142. BenEzra D, Cohen E, Chajek T, Friedman G, Pizanti S, de Courten C, Harris W (1988) Evaluation of conventional therapy versus cyclosporine A in Behçet's syndrome. Transplant Proc 20:136-143.

143. Masuda K, Nakajima A, Urayama A, Nakae K, Kogure M, Inaba G (1989) Double-masked trial of cyclosporin versus colchicine and long-term open study of cyclosporin in Behçet's disease. Lancet 1:1093-1096.

144. Yamada Y, Sugita S, Tanaka H, Kamoi K, Kawaguchi T, Mochizuki M (2010) Comparison of infliximab versus ciclosporin during the initial 6-month treatment period in Behcet disease. Br J Ophthalmol 94:284-288.

145. Yazici H, Ozyazgan Y (1999) Medical management of Behçet's syndrome. Dev Ophthalmol 31:118-131.

146. Ergun T, Gürbüz O, Yurdakul S, Hamuryudan V, Bekiroğlu N, Yazici H (1997) Topical cyclosporine-A for treatment of oral ulcers of Behçet's syndrome. Int J Dermatol 36:720.

147. Cantini F, Salvarani C, Niccoli L, Padula A, Arena AI, Bellandi F, Macchioni P, Olivieri I (1999) Treatment of thrombophlebitis of Behçet's disease with low dose cyclosporin A. Clin Exp Rheumatol 17:391-392.

148. Avci O, Gürler N, Güneş AT (1997) Efficacy of cyclosporine on mucocutaneous manifestations of Behçet's disease. J Am Acad Dermatol 36:796-797.

149. Kotake S, Higashi K, Yoshikawa K, Sasamoto Y, Okamoto T, Matsuda H (1999) Central nervous system symptoms in patients with Behçet disease receiving cyclosporine therapy. Ophthalmology 106:586-589.

150. Kötter I, Günaydin I, Batra M, Vonthein R, Stübiger N, Fierlbeck G, Melms A (2005) CNS involvement occurs more frequently in patients with Behçet's disease under cyclosporin A (CSA) than under other medications - results of a retrospective analysis of 117 cases. Clin Rheumatol 25:482-486.

151. Kato Y, Numaga J, Kato S, Kaburaki T, Kawashima H, Fujino Y (2001) Central nervous system symptoms in a population of Behçet's disease patients with refractory uveitis treated with cyclosporine A. Clin Experiment Ophthalmol 29:335-336.

152. Jamilloux Y, Gerfaud-Valentin M, Henry T, Sève P (2015) Treatment of adult-onset Still's disease: a review. Ther Clin Risk Manag 11:33-43.

153. Mitamura M, Tada Y, Koarada S, Noue H, Suematsu R, Ohta A, Nagasawa K (2009) Cyclosporin A treatment for Japanese patients with severe adult-onset Still's disease. Mod Rheumatol 19:57-63.

154. Efthimiou P, Kadavath S, Mehta B (2014) Life-threatening complications of adult-onset Still's disease. Clin Rheumatol 33:305-314. 
155. Mori T, Tanigawa M, Iwasaki E, Tamaki S, Ono T, Wada H, Deguchi K, Shirakawa S (1993) Cyclosporine therapy of adult onset Still's disease with disseminated intravascular coagulation. Rinsho Ketsueki 34:147-152.

156. Park J-H, Bae JH, Choi Y-S, Lee HS, Jun JB, Jung S, Yoo DH, Bae SC, Kim TH (2004) Adult-onset Still's disease with disseminated intravascular coagulation and multiple organ dysfunctions dramatically treated with cyclosporine A. J Korean Med Sci 19:137-141.

157. Hamidou M, Boutoille D, Masseau A, Garand R, Raffi F (2005) Adult-onset Still disease with hemophagocytic syndrome treated with cyclosporine. Presse Med 34:1634-1636.

158. Mizrahi M, Ben-Chetrit E (2009) Relapsing macrophage activating syndrome in a 15-yearold girl with Still's disease: a case report. J Med Case Rep 3:138.

159. Her M-Y, Kim T-H, Chang H-K, Lee W-S, Yoo D-H (2007) Successful treatment of acquired amegakaryocytic thrombocytopenia with cyclosporine in adult onset Still's disease. Rheumatol Int 27:295-298.

160. Nagashima T, Aoki Y, Onishi S, Iwamoto M, Okazaki H, Minota S (2008) Steroidrefractory severe hepatic failure in adult onset Still's disease responding to cyclosporine. Clin Rheumatol 27:1451-1453.

161. Kunert KS, Tisdale AS, Gipson IK (2002) Goblet cell numbers and epithelial proliferation in the conjunctiva of patients with dry eye syndrome treated with cyclosporine. Arch Ophthalmol 120:330-337.

162. Kunert KS, Tisdale AS, Stern ME, Smith JA, Gipson IK (2000) Analysis of topical cyclosporine treatment of patients with dry eye syndrome: effect on conjunctival lymphocytes. Arch Ophthalmol 118:1489-1496.

163. Pflugfelder SC, De Paiva CS, Villarreal AL, Stern ME (2008) Effects of sequential artificial tear and cyclosporine emulsion therapy on conjunctival goblet cell density and transforming growth factor-beta2 production. Cornea 27:64-69.

164. Foulks GN (2006) Topical cyclosporine for treatment of ocular surface disease. Int Ophthalmol Clin 46:105-122.

165. Stevenson D, Tauber J, Reis BL (2000) Efficacy and safety of cyclosporin A ophthalmic emulsion in the treatment of moderate-to-severe dry eye disease: a dose-ranging, randomized trial. The Cyclosporin A Phase 2 Study Group. Ophthalmology 7:967-974.

166. Barber LD, Pflugfelder SC, Tauber J, Foulks GN (2005) Phase III safety evaluation of cyclosporine $0.1 \%$ ophthalmic emulsion administered twice daily to dry eye disease patients for up to 3 years. Ophthalmology 112:1790-1794.

167. Sall K, Stevenson OD, Mundorf TK, Reis BL (2000) Two multicenter, randomized studies of the efficacy and safety of cyclosporine ophthalmic emulsion in moderate to severe dry eye disease. CsA Phase 3 Study Group. Ophthalmology 107:631-639.

168. Roberts CW, Carniglia PE, Brazzo BG (2007) Comparison of topical cyclosporine, punctal occlusion, and a combination for the treatment of dry eye. Cornea 26:805-809.

169. Kim EC, Choi J-S, Joo C-K (2009) A comparison of vitamin a and cyclosporine a $0.05 \%$ 
eye drops for treatment of dry eye syndrome. Am J Ophthalmol 147:206-213.e3.

170. Drosos AA, Skopouli FN, Costopoulos JS, Papadimitriou CS, Moutsopoulos HM (1986) Cyclosporin A (CyA) in primary Sjögren's syndrome: a double blind study. Ann Rheum Dis 45:732-735.

171. Drosos AA, Skopouli FN, Galanopoulou VK, Kitridou RC, Moutsopoulos HM (1986) Cyclosporin a therapy in patients with primary Sjögren's syndrome: results at one year. Scand J Rheumatol 61:246-249.

172. Heckmatt J, Hasson N, Saunders C, Thompson N, Peters AM, Cambridge G, Rose M, Hyde SA, Dubowitz V (1989) Cyclosporin in juvenile dermatomyositis. Lancet 8646:10631066.

173. Reiff A, Rawlings DJ, Shaham B, Franke E, Richardson L, Szer IS, Bernstein BH (1997) Preliminary evidence for cyclosporin A as an alternative in the treatment of recalcitrant juvenile rheumatoid arthritis and juvenile dermatomyositis. J Rheumatol 24:2436-2443.

174. Pistoia V, Buoncompagni A, Scribanis R, Fasce L, Alpigiani G, Cordone G, Ferrarini M, Borrone C, Cottafava F (1993) Cyclosporin A in the treatment of juvenile chronic arthritis and childhood polymyositis-dermatomyositis. Results of a preliminary study. Clin Exp Rheumatol 11:203-208

175. Ruperto N, Pistorio A, Knupp Feitosa de Oliveira S, Cuttica R, Ravelli A, Fischbach M, et al (2013) A randomized trial in new onset juvenile dermatomyositis: prednisone versus prednisone plus cyclosporine versus prednisone plus methotrexate. Pediatr Rheumatol 11:019.

176. Ringold S, Weiss PF, Beukelman T, DeWitt EM, Ilowite NT, Kimura Y, Laxer RM, Lovell DJ, Nigrovic PA, Robinson AB, Vehe RK; American College of Rheumatology (2013) 2013 update of the 2011 American College of Rheumatology recommendations for the treatment of juvenile idiopathic arthritis: recommendations for the medical therapy of children with systemic juvenile idiopathic arthritis and tuberculosis screening among children receiving biologic medications. Arthritis Rheum 65:2499-2512.

177. Gerloni V, Cimaz R, Gattinara M, Arnoldi C, Pontikaki I, Fantini F (2001) Efficacy and safety profile of cyclosporin $\mathrm{A}$ in the treatment of juvenile chronic (idiopathic) arthritis. Results of a 10-year prospective study. Rheumatology (Oxford) 40:907-913

178. Ostensen M, Høyeraal HM, Kåss E (1988) Tolerance of cyclosporine A in children with 17refractory juvenile rheumatoid arthritis. J Rheumatol 15:1536-1538.

179. Ravelli A, Moretti C, Temporini F, Rossi F, Magni-Manzoni S, Pistorio A, Martini A (2002) Combination therapy with methotrexate and cyclosporine A in juvenile idiopathic arthritis. Clin Exp Rheumatol 20:569-572.

180. Mouy R, Stephan JL, Pillet P, Haddad E, Hubert P, Prieur AM (1996) Efficacy of cyclosporine $\mathrm{A}$ in the treatment of macrophage activation syndrome in juvenile arthritis: report of five cases. J Pediatr 129:750-754.

181. Ravelli A, De Benedetti F, Viola S, Martini A (1996) Macrophage activation syndrome in systemic juvenile rheumatoid arthritis successfully treated with cyclosporine. J Pediatr 128:275-278. 
182. Stephan JL, Koné-Paut I, Galambrun C, Mouy R, Bader-Meunier B, Prieur AM (2001) Reactive haemophagocytic syndrome in children with inflammatory disorders. A retrospective study of 24 patients. Rheumatology (Oxford) 40:1285 - 1292.

183. Sawhney S, Woo P, Murray KJ (2001) Macrophage activation syndrome: a potentially fatal complication of rheumatic disorders. Arch Dis Child 85:421-426.

184. Kounami S, Yoshiyama M, Nakayama K, Okuda M, Okuda S, Aoyagi N, Yoshikawa N (2005) Macrophage activation syndrome in children with systemic-onset juvenile chronic arthritis. Acta Haematol 113:124-129.

185. Cortis E, Insalaco A (2006) Macrophage activation syndrome in juvenile idiopathic arthritis. Acta Paediatr 95:38-41.

186. You CR, Kim HR, Yoon CH, Lee SH, Park SH, Kim HY (2006) Macrophage activation syndrome in juvenile rheumatoid arthritis successfully treated with cyclosporine A: a case report. J Korean Med Sci 21:1124-1127.

187. Lin CI, Yu HH, Lee JH, Wang LC, Lin YT, Yang YH, Chiang BL (2012) Clinical analysis of macrophage activation syndrome in pediatric patients with autoimmune diseases. Clin Rheumatol 31:1223-1230.

188. Ishii N, Watashi K, Hishiki T, Goto K, Inoue D, Hijikata M, Wakita T, Kato N, Shimotohno K (2006) Diverse Effects of Cyclosporine on Hepatitis C Virus Strain Replication. J Virol 80:4510-4520.

189. Nakagawa M, Sakamoto N, Enomoto N, Tanabe Y, Kanazawa N, Koyama T, Kurosaki M, Maekawa S, Yamashiro T, Chen CH, Itsui Y, Kakinuma S, Watanabe M (2004) Specific inhibition of hepatitis $\mathrm{C}$ virus replication by cyclosporin A. Biochem Biophys Res Commun 313:42-47.

190. Galeazzi M, Giannitti C, Manganelli S, Benucci M, Scarpato S, Bazzani C, Caporali R, Sebastiani GD (2008) Treatment of rheumatic diseases in patients with HCV and HIV infection. Autoimmun Rev 8:100-103.

191. Kohjima M, Enjoji M, Higuchi N, Kotoh K, Kato M, Takayanagi R, Nakamuta M (2007) NIM811, a nonimmunosuppressive cyclosporine analogue, suppresses collagen production and enhances collagenase activity in hepatic stellate cells. Liver Int 27:1273-1281.

192. Galeazzi M, Bellisai F, Giannitti C, Manganelli S, Morozzi G, Sebastiani GD (2007) Safety of Cyclosporin A in HCV-Infected Patients: Experience with Cyclosporin A in Patients Affected by Rheumatological Disorders and Concomitant HCV Infection. Annals N Y Acad Sci 1110:544-549.

193. Galeazzi M, Bellisai F, Manganelli S, Morozzi G, Sebastiani GD (2006) Cyclosporine A for the treatment of autoimmune disorders in $\mathrm{HCV}$ infected patients. Autoimmun Rev 5:493-498.

194. Bellisai F, Giannitti C, Donvito A, Galeazzi M (2006) Combination therapy with cyclosporine A and anti-TNF- $\alpha$ agents in the treatment of rheumatoid arthritis and concomitant hepatitis C virus infection. Clin Rheumatol 26:1127-1129.

195. Tendron-Franzin A, Gouyon J-B, Guignard J-P, Decramer S, Justrabo E, Gilbert T, 
Semama DS (2004) Long-term effects of in utero exposure to cyclosporin A on renal function in the rabbit. J Am Soc Nephrol 15:2687-2693.

196. Bar Oz B, Hackman R, Einarson T, Koren G (2001) Pregnancy outcome after cyclosporine therapy during pregnancy: a meta-analysis. Transplantation 71:1051-1055.

197. Gerosa M, Meroni PL, Cimaz R (2014) Safety considerations when prescribing immunosuppression medication to pregnant women. Expert Opin Drug Saf 13:1591-9.

198. Stanley CW, Gottlieb R, Zager R, Eisenberg J, Richmond R, Moritz MJ, Armenti VT (1999) Developmental well-being in offspring of women receiving cyclosporine post-renal transplant. Transplant Proc 31:241-2.

199. Cimaz R, Meregalli E, Biggioggero M, Borghi O, Tincani A, Motta M, Airò P, Meroni PL (2004) Alterations in the immune system of children from mothers treated with immunosuppressive agents during pregnancy. Toxicol Lett 149:155-162.

200. Biggioggero M, Borghi MO, Gerosa M, Trespidi L, Cimaz R, Meroni PL (2007) Immune function in children born to mothers with autoimmune diseases and exposed in utero to immunosuppressants. Lupus 16:651-656.

201. Moretti ME, Sgro M, Johnson DW, Sauve RS, Woolgar MJ, Taddio A, Verjee Z, Giesbrecht E, Koren G, Ito S (2003) Cyclosporine excretion into breast milk. Transplantation 75:2144-2146.

202. Nyberg G, Haljamäe U, Frisenette-Fich C, Wennergren M, Kjellmer I (1998) Breastfeeding during treatment with cyclosporine. Transplantation 65:253-255.

203. American Academy of Pediatrics Committee on Drugs (2001) Transfer of drugs and other chemicals into human milk. Pediatrics 108:776-789.

204. Borigini MJ, Paulus HE (1995) Innovative treatment approaches for rheumatoid arthritis. Combination therapy. Baillieres Clin Rheumatol 9:689-710.

205. Griffiths B, Emery P (2001) The treatment of lupus with cyclosporin A. Lupus 10:165-170.

206. Ryan C, Amor KT, Menter A (2010) The use of cyclosporine in dermatology: Part II. J Am Acad Dermatol 63:949-972.

207. U.S. Food and Drug Administration Web site.

http://www.accessdata.fda.gov/drugsatfda_docs/label/2009/050715s027,050716s0281bl.pdf

Gough A, Helliwell P, Martin M, Huston G, Pease C, Veale DJ, Isaacs J, van der Heijde DM, Emery P (2000) Treatment of poor-prognosis early rheumatoid arthritis. A randomized study of treatment with methotrexate, cyclosporin A, and intraarticular corticosteroids compared with sulfasalazine alone. Arthritis Rheum 43:1809-19.

209. Bejarano V, Conaghan PG, Proudman SM, Buch MH, Brown AK, Emery P (2009) Longterm efficacy and toxicity of ciclosporin $A$ in combination with methotrexate in poor prognosis rheumatoid arthritis. Ann Rheum Dis 68:761-763.

210. Sugiyama M, Ogasawara H, Kaneko H, Hishikawa T, Sekigawa I, Iida N, Hashimoto H, Hirose S (1998) Effect of extremely low dose cyclosporine treatment on the 
thrombocytopenia in systemic lupus erythematosus. Lupus 7:53-6.

211. Bambauer R, Schwarze U, Schiel R (2000) Cyclosporin A and therapeutic plasma exchange in the treatment of severe systemic lupus erythematosus. Artif Organs 24:852856.

212. Morton SJ, Powell RJ (2000) Cyclosporin and tacrolimus: their use in a routine clinical setting for scleroderma. Rheumatology (Oxford) 39:865-869.

213. Kim H-J, Hong Y-K, Yoo W-H (2009) Dermatomyositis, complicated with pneumomediastinum, successfully treated with cyclosporine A: a case report and review of literature. Rheumatol Int 29:1101-1104.

214. Kotani T, Takeuchi T, Makino S, Hata K, Yoshida S, Nagai K, Wakura D, Shoda T, Hanafusa $T$ (2011) Combination with corticosteroids and cyclosporin-A improves pulmonary function test results and chest HRCT findings in dermatomyositis patients with acute/subacute interstitial pneumonia. Clin Rheumatol. 30:1021-1028. 